\title{
Evidence for inflation in an axion landscape
}

\author{
Pran Nath and Maksim Piskunov \\ Department of Physics, Northeastern University, \\ Boston, MA 02115-5000, U.S.A. \\ E-mail: p.nath@northeastern.edu, m.piskunov@northeastern.edu
}

ABSTRACT: We discuss inflation models within supersymmetry and supergravity frameworks with a landscape of chiral superfields and one $\mathrm{U}(1)$ shift symmetry which is broken by non-perturbative symmetry breaking terms in the superpotential. We label the pseudo scalar component of the chiral fields axions and their real parts saxions. Thus in the models only one combination of axions will be a pseudo-Nambu-Goldstone-boson which will act as the inflaton. The proposed models constitute consistent inflation for the following reasons: the inflation potential arises dynamically with stabilized saxions, the axion decay constant can lie in the sub-Planckian region, and consistency with the Planck data is achieved. The axion landscape consisting of $m$ axion pairs is assumed with the axions in each pair having opposite charges. A fast roll-slow roll splitting mechanism for the axion potential is proposed which is realized with a special choice of the axion basis. In this basis the $2 m$ coupled equations split into $2 m-1$ equations which enter in the fast roll and there is one unique linear combination of the $2 m$ fields which controls the slow roll and thus the power spectrum of curvature and tensor perturbations. It is shown that a significant part of the parameter space exists where inflation is successful, i.e., $N_{\text {pivot }}=[50,60]$, the spectral index $n_{s}$ of curvature perturbations, and the ratio $r$ of the power spectrum of tensor perturbations and curvature perturbations, lie in the experimentally allowed regions given by the Planck experiment. Further, it is shown that the model allows for a significant region of the parameter space where the effective axion decay constant can lie in the sub-Planckian domain. An analysis of the tensor spectral index $n_{t}$ is also given and the future experimental data which constraints $n_{t}$ will further narrow down the parameter space of the proposed inflationary models. Topics of further interest include implications of the model for gravitational waves and non-Gaussianities in the curvature perturbations. Also of interest is embedding of the model in strings which are expected to possess a large axionic landscape.

KEYWORDs: Phenomenological Models, Supersymmetry Phenomenology

ArXiv EPrint: 1712.01357 


\section{Contents}

1 Introduction 1

2 A supersymmetric model of inflation for an axionic landscape 3

3 The axion landscape and a fast roll-slow roll splitting mechanism 5

4 Extension to supergravity $\quad 8$

5 Consistency with Planck data $[14,15] \quad 9$

5.1 Experimental observables 9

$\begin{array}{ll}\text { 5.2 Monte Carlo analysis for fit to data } & 11\end{array}$

$\begin{array}{ll}\text { 5.3 Generation of a flat inflation potential } & 18\end{array}$

6 Conclusion $\quad 19$

$\begin{array}{ll}\text { A Preliminaries } & 20\end{array}$

$\begin{array}{ll}\text { B Power spectrum and spectral indices } & 21\end{array}$

C Emergence of a flat inflation potential for axions 23

\section{Introduction}

Inflationary models resolve a number of problems associated with Big Bang cosmology which include the flatness problem, the horizon problem, and the monopole problem [1-6] (for a review see [7] and for effective field theory of inflation see [8]). In inflation models quantum fluctuations at the time of horizon exit carry significant information regarding the characteristics of the inflationary model [9-13]. The cosmic microwave background (CMB) radiation anisotropy allows extraction of such characteristics which can discriminate among models. Thus recently the astrophysical data from the Planck experiment [14-16] has put significant constraints on models eliminating many and reducing the parameter space of others. One class of models are those associated with the so called natural inflation where the inflaton is an axionic field. Thus natural inflation is described by a simple potential $[17,18]$

$$
V(a)=\Lambda^{4}\left(1+\cos \left(\frac{a}{f}\right)\right)
$$

where $a$ is the axion field and $f$ is the axion decay constant. The Planck data requires $f$ significantly greater $M_{P l}$, i.e., $f>10 M_{P l}$ where $M_{P l}$ is the Planck mass. Now $f>M_{P l}$ 
is undesirable since a global symmetry is not preserved by quantum gravity unless it has a gauge origin. Further, string theory prefers $f$ below $M_{P L}[19,20]$. Reduction of the axion decay constant turns out to be a significant problem and various procedures have been pursued to overcome it. These include the so called alignment mechanism [21, 22], the two axion Dante's inferno model [23] and N-flation [24-32]. Other models using shift symmetry include [33-36] (for a review and a more extensive set of references of axionic inflation and of axionic cosmology see [37, 38].).

In this work we introduce an inflation model in an axion landscape with a U(1) symmetry and with $m$ pairs of chiral fields where the chiral fields in each pair are oppositely charged under the same U(1) global symmetry. We wish to note here that different authors define the term "axion" differently. In the analysis here we will use the term "axion" for the pseudo-scalar component of any chiral field and the corresponding real part will be called a "saxion". In our analysis we have only one U(1) global symmetry and thus breaking of it would lead to only one pseudo-Nambu-Goldstone-boson (PNGB) and the remaining pseudo-scalars are not PNGBs. It is important to keep this distinction in mind since sometimes the term "axion" is automatically interpreted as being a PNGB which is not the case in the analysis here. Returning to the construction of our model the superpotential is chosen to consist of two parts where one part is invariant under the $\mathrm{U}(1)$ symmetry and the other consists of a symmetry breaking piece such as the one arising from instanton effects. Here we show that a fast roll-slow roll splitting mechanism exits which allows a decomposition of the axion potential into a fast roll and a slow roll part where inflation is driven by the slow roll part. This set up reduces a multi-field coupled axion system with $2 m$ axions to an effective single axion field potential which controls inflation. Using this set up we analyze both supersymmetry and supergravity models and show that under the constraints of stabilized saxions, one can find inflation models with the axion decay constant $f<M_{P l}$ consistent with the data from the Planck experiment.

The outline of the rest of the paper is as follows. In section 2, we describe the supersymmetric model of inflation for an axionic landscape consisting of $m$ pairs of axions with each pair oppositely charged under the $\mathrm{U}(1)$ symmetry. The superpotential consists of two terms: a part which is invariant under the $\mathrm{U}(1)$ symmetry and a part which breaks it arising possibly from instantons. Using this superpotential we deduce the conditions for stabilized saxions and then compute the scalar potential for the axions under the constraints of stabilized saxions. In this section we also compute the axion mass matrices. In section 3 , we carry out a decomposition of the scalar axion potential into a fast roll and a slow part. To accomplish this we first find the basis where $2 m-1$ axions are heavy and one axion is light, i.,e., massless in the limit when there is no breaking of the shift symmetry. Part of the potential which contains the heavy fields produces the fast roll inflation while the part that contains the light field generates the slow roll part. The decomposition reduces the multi-field inflation to a single field inflation. Here we also show that in the slow roll part an effective axion decay constant $f_{e}$ enters which is given by $f_{e}=\sqrt{2 m} f$ where $f$ is the common decay constant of the axions that enter the superpotential. This result was first derived in [24] but here we give a more general derivation of it. In section 4, we extend the analysis of section 3 to supergravity with similar conclusions. In section 5 , we give an 
analysis of the number of e-foldings, of the power spectrum for curvature and tensor perturbations, and of the scalar and of the tensor spectral indices. Specifically we show that much of the allowed parameter space of experiment is accessible in this model and future experiment will constrain the model more stringently. In this section we also show how the cosine functions generate a locally flat potential necessary for inflation. Conclusions are given in section 6 . In appendices $\mathrm{A}$ and $\mathrm{B}$ we define notation and give some mathematical background useful for the analysis carried out in section 5. In appendix $\mathrm{C}$ we illustrate the emergence of a flat inflation potential arising from the superposition of cosine functions.

\section{A supersymmetric model of inflation for an axionic landscape}

We discuss here a general supersymmetric framework for axionic inflation to occur. ${ }^{1}$ The axions we consider are not QCD axions [68-70] which were originally the basis of the analysis of [17]. In string theory axions occur which are not related to the QCD axions [20, 71]. Thus we consider the existence of a shift symmetry and assume that in an axionic landscape, such as the one that one might expect in string theory, there are a number of axionic fields carrying the same U(1) quantum number. Now suppose we have a set of fields $S_{i}(i=1, \cdots, m)$ where $S_{i}$ carry the same charge under the shift symmetry and the fields $\bar{S}_{i}(i=1, \cdots, m)$ carry the opposite charge. Thus under $\mathrm{U}(1)$ transformations one has

$$
S_{i} \rightarrow e^{i q \lambda} S_{i}, \quad \bar{S}_{i} \rightarrow e^{-i q \lambda} \bar{S}_{i}, \quad i=1, \cdots, m
$$

The superfields $S_{i}$ have an expansion,

$$
S_{i}=\phi_{i}+\theta \chi_{i}+\theta \theta F_{i},
$$

where $\phi_{i}$ is a complex scalar field consisting of the saxion (the real part) and the axion (the imaginary part), $\chi_{i}$ is the axino, and $F_{i}$ is an auxiliary field. Similarly the superfields $\bar{S}_{i}$ have an expansion: $\bar{S}_{i}=\bar{\phi}_{i}+\bar{\theta} \bar{\xi}_{i}+\bar{\theta} \bar{\theta} \bar{F}_{i}$. We now consider a superpotential of the form

$$
W=W_{s}(S, \bar{S})+W_{s b}(S, \bar{S}),
$$

where $W_{s}$ is the part that depends on the fields $S_{i}, \bar{S}_{i}$ and is invariant under the shift symmetry. $W_{s b}$ is a part which breaks the shift symmetry and has the form

$$
W_{s b}=\sum_{i} A_{i}(S, \bar{S}) e^{-T_{i}}
$$

where $T_{i}$ is the action of the $i$-th instanton. In general gauge invariance and holomorphy allow non-perturbative terms of the type

$$
A \frac{S^{n}}{M_{P}^{n-3}} e^{-T}, \quad \bar{A} \frac{\bar{S}^{n}}{M_{P}^{n-3}} e^{-T}
$$

\footnotetext{
${ }^{1}$ For references to early work in supersymmetry and supergravity see [39-67].
} 
Detailed structure will depend on the instanton zero modes (see [71, 72] and the references there in). Thus we assume the following forms for $W(S, \bar{S}),{ }^{2}$

$$
W(S, \bar{S})=\sum_{k=1}^{m} \sum_{l=1}^{m}\left(\mu_{k l} S_{k} \bar{S}_{l}+\frac{\lambda_{k l}}{2 M}\left(S_{k} \bar{S}_{l}\right)^{2}\right)+\sum_{k=1}^{m} \sum_{l=1}^{q} A_{k l} S_{k}^{l}+\sum_{k=1}^{m} \sum_{l=1}^{q} \bar{A}_{k l} \bar{S}_{k}^{l} .
$$

Here the terms in the first brace on the right hand side are invariant under the shift symmetry while the remaining terms on the right hand side violate the shift symmetry. The variation of the superpotential with respect to $\phi_{k}$ and $\bar{\phi}_{k}$ generate the constraints that determine the VEVs of $\phi_{k}$ and $\bar{\phi}_{k}$. We assume CP conserving vacua so that the VEVs of the CP odd axionic fields vanish while we set $f_{k}=<\phi_{k}>$ and $\bar{f}_{k}=<\bar{\phi}_{k}>$. The constraint equations arising from the variation of the superpotential with respect to $\phi_{k}$ and $\bar{\phi}_{k}$ are

$$
\begin{aligned}
& \frac{\partial W(\phi, \bar{\phi})}{\partial \phi_{k}}=0, \\
& \frac{\partial W(\phi, \bar{\phi})}{\partial \bar{\phi}_{k}}=0 .
\end{aligned}
$$

We may parametrize $\phi_{k}$ and $\bar{\phi}_{k}$ so that

$$
\phi_{k}=\left(f_{k}+\rho_{k}\right) e^{i a_{k} / f_{k}}, \quad \bar{\phi}_{k}=\left(\bar{f}_{k}+\bar{\rho}_{k}\right) e^{i \bar{a}_{k} / \bar{f}_{k}},
$$

where $f_{k}=<\phi_{k}>, \bar{f}_{k}=<\bar{\phi}_{k}>$ and $\left(\rho_{k}, a_{k}\right)$ and $\left(\bar{\rho}_{k}, \bar{a}_{k}\right)$ are the fluctuations of the quantum fields around their vacuum expectation values $f_{k}\left(\bar{f}_{k}\right)$. They are constrained by the stability conditions for the saxions eqs. (2.7) which give

$$
\begin{aligned}
& \sum_{l=1}^{m}\left(\mu_{k l} \bar{f}_{l}+\frac{\lambda_{k l}}{M} f_{k} \bar{f}_{l}^{2}\right)+\sum_{l=1}^{q} l A_{k l} f_{k}^{l-1}=0, \\
& \sum_{l=1}^{m}\left(\mu_{l k} f_{l}+\frac{\lambda_{l k}}{M} f_{l}^{2} \bar{f}_{k}\right)+\sum_{l=1}^{q} l \bar{A}_{k l} \bar{f}_{k}^{l-1}=0 .
\end{aligned}
$$

We focus here on the scalar potential for the axions and thus we expand around the minimum of the potential of the saxions, i.e., we set $\rho_{k}=0=\bar{\rho}_{k}$. Using the saxion stability conditions given by eq. (2.9) a somewhat lengthy computation gives for the scalar potential

$$
V(a, \bar{a})=V_{s}(a, \bar{a})+V_{s b}(a, \bar{a}),
$$

where

$$
\begin{aligned}
V_{s}(a, \bar{a})= & \sum_{k=1}^{m}\left(\sum _ { l = 1 } ^ { m } \sum _ { p = 1 } ^ { m } \left(\mu_{k l} \mu_{k p} \bar{f}_{l} \bar{f}_{p} \cos \left(\frac{\bar{a}_{p}}{\bar{f}_{p}}-\frac{\bar{a}_{l}}{\bar{f}_{l}}\right)+\mu_{l k} \mu_{p k} f_{l} f_{p} \cos \left(\frac{a_{p}}{f_{p}}-\frac{a_{l}}{f_{l}}\right)\right.\right. \\
& +\frac{\lambda_{k l}}{M} \frac{\lambda_{k p}}{M} f_{k}^{2} \bar{f}_{l}^{2} \bar{f}_{p}^{2} \cos \left(2 \frac{\bar{a}_{p}}{\bar{f}_{p}}-2 \frac{\bar{a}_{l}}{\bar{f}_{l}}\right)+\frac{\lambda_{l k}}{M} \frac{\lambda_{p k}}{M} f_{l}^{2} f_{p}^{2} \bar{f}_{k}^{2} \cos \left(2 \frac{a_{p}}{f_{p}}-2 \frac{a_{l}}{f_{l}}\right) \\
& \left.+2 \mu_{k l} \frac{\lambda_{k p}}{M} f_{k} \bar{f}_{l} \bar{f}_{p}^{2} \cos \left(\frac{a_{k}}{f_{k}}+2 \frac{\bar{a}_{p}}{\bar{f}_{p}}-\frac{\bar{a}_{l}}{\bar{f}_{l}}\right)+2 \mu_{l k} \frac{\lambda_{p k}}{M} \bar{f}_{k} f_{l} f_{p}^{2} \cos \left(\frac{\bar{a}_{k}}{\bar{f}_{k}}+2 \frac{a_{p}}{f_{p}}-\frac{a_{l}}{f_{l}}\right)\right),
\end{aligned}
$$

\footnotetext{
${ }^{2} \mathrm{~A}$ simplified version of this form of the superpotential has been considered recently in the context of an ultralight axion [71].
} 
and

$$
\begin{aligned}
V_{s b}(a, \bar{a})= & \sum_{l=1}^{m} \sum_{r=1}^{q}\left(2 r A_{k r} \mu_{k l} f_{k}^{r-1} \bar{f}_{l} \cos \left((r-1) \frac{a_{k}}{f_{k}}-\frac{\bar{a}_{l}}{\bar{f}_{l}}\right)\right. \\
& +2 r \bar{A}_{k r} \mu_{l k} \bar{f}_{k}^{r-1} f_{l} \cos \left((r-1) \frac{\bar{a}_{k}}{\bar{f}_{k}}-\frac{a_{l}}{f_{l}}\right) \\
& \left.+2 r A_{k r} \frac{\lambda_{k l}}{M} f_{k}^{r} \bar{f}_{l}^{2} \cos \left((r-2) \frac{a_{k}}{f_{k}}-2 \frac{\bar{a}_{l}}{\bar{f}_{l}}\right)+2 r \bar{A}_{k r} \frac{\lambda_{l k}}{M} \bar{f}_{k}^{r} f_{l}^{2} \cos \left((r-2) \frac{\bar{a}_{k}}{\bar{f}_{k}}-2 \frac{a_{l}}{f_{l}}\right)\right) \\
& \left.+\sum_{l=1}^{q} \sum_{r=1}^{q}\left(l r A_{k l} A_{k r} f_{k}^{l+r-2} \cos \left((r-l) \frac{a_{k}}{f_{k}}\right)+\operatorname{lr} \bar{A}_{k l} \bar{A}_{k r} \bar{f}_{k}^{l+r-2} \cos \left((r-l) \frac{\bar{a}_{k}}{\bar{f}_{k}}\right)\right)\right) .
\end{aligned}
$$

Here $V_{s}$ is part of the potential that preserves the shift symmetry and $V_{s b}$ is the part that breaks the shift symmetry. We note that because of the periodic nature of the potential, eqs. (2.10)-(2.12) provide a valid theory even for $a_{i}>M_{P l}$.

Next we look at the mass matrix for the axions. The mass matrix consists of three types of terms: $M\left(a_{l}, a_{p}\right)$ which involves only the axions $a_{i}, i=1, \cdots, m, M\left(\bar{a}_{l}, \bar{a}_{p}\right)$ which involves only the axions $\bar{a}_{i}, i=1, \cdots, m$, and the matrix with the cross terms $M\left(a_{k}, \bar{a}_{p}\right)$. For the computation of the heavier axion masses it is sufficient to look at the mass matrix in the limit when shift symmetry breaking is absent, i.e., ignore $V_{s b}$, and inclusion of the breaking of the shift symmetry would make only negligible contribution to the heavy axion masses. An explicit computation of these gives the following: for $M\left(a_{l}, a_{p}\right)$ one has

$$
M\left(a_{l}, a_{p}\right)=2 \delta_{l p} \frac{1}{f_{p}^{2}}\left(\sum_{k=1}^{m} \mu_{p k} \bar{f}_{k}\right)^{2}+2 \sum_{k=1}^{m}\left(\mu_{l k}+2 \frac{\lambda_{l k}}{M} \bar{f}_{k} f_{l}\right)\left(\mu_{p k}+2 \frac{\lambda_{p k}}{M} \bar{f}_{k} f_{p}\right) .
$$

For $M\left(\bar{a}_{l}, \bar{a}_{p}\right)$ one has

$$
M\left(\bar{a}_{l}, \bar{a}_{p}\right)=2 \delta_{l p} \frac{1}{\bar{f}_{p}^{2}}\left(\sum_{k=1}^{m} \mu_{k p} f_{k}\right)^{2}+2 \sum_{k=1}^{m}\left(\mu_{k l}+2 \frac{\lambda_{k l}}{M} f_{k} \bar{f}_{l}\right)\left(\mu_{k p}+2 \frac{\lambda_{k p}}{M} f_{k} \bar{f}_{p}\right),
$$

and for the cross term $M\left(a_{k}, \bar{a}_{p}\right)$ one has

$$
M\left(a_{k}, \bar{a}_{p}\right)=M\left(\bar{a}_{p}, a_{k}\right)=-2 \sum_{l=1}^{m}\left(2 \frac{\lambda_{k p}}{M}\left(\mu_{k l} \bar{f}_{l} \bar{f}_{p}+\mu_{l p} f_{l} f_{k}\right)-\mu_{k p}\left(\frac{\lambda_{k l}}{M} \bar{f}_{l}^{2}+\frac{\lambda_{l p}}{M} f_{l}^{2}\right)\right)
$$

This mass matrix is $2 m \times 2 m$ dimensional. It has $2 m-1$ non-zero eigenvalues and one eigenvalue is identically zero which corresponds to the inflaton. This result is a consequence of the Goldstone theorem [73-75] which requires that the spontaneous breaking of a single global U(1) symmetry leads to a single massless Goldstone mode which implies that there is just one possibility for the inflation field which ia the zero mode in the diagonalization of a $2 m \times 2 m$ matrix as noted above and all other modes are heavy after spontaneous breaking.

\section{The axion landscape and a fast roll-slow roll splitting mechanism}

As discussed in the preceding section, even though we have a landscape of axions, i.e., pseudo scalar fields, there is only one $\mathrm{U}(1)$ shift symmetry and correspondingly there is 
only one linear combination of the axion fields which is the pseudo-Nambu-Goldstone-boson and acts as the inflation field. The reduction of the multi axion system to a single inflation field does not mean that one has the same dynamics if one started with a single field. The reason for this is the follows: our starting point was a landscape of axions each of which undergoes a shift under the same U(1). The inflation field thus includes pieces of each of these fields. Further, although in the limit of no breaking of the shift symmetry, the dynamics of the (pNGB) inflaton is decoupled from the rest of the axions, there is mixing between the two sectors once the shift symmetry is broken. In this case the diagonalization will yield $2 m-1$ massive axions and a relatively lighter inflation field. In principle all the axions both light and heavy enter in inflation, except that the $2 m-1$ axions produce a fast roll and die off relatively quickly while the inflation field produces the slow roll. To check that the multi-field analysis is faithfully reproduced by the effective single field, we carry out a numerical analysis for a multi-field case and compare the result to that for the effective single field and find that the fast roll-slow roll decomposition is justified. We emphasize that the assumption of only one global symmetry will automatically result in only one inflation direction and one inflation candidate and all other axions will be heavy. After the breaking of the shift symmetry, the potential for the axions will in general be mixed involving all the axions. We discuss below the explicit procedure for the splitting of the total potential given by eqs. (2.10)-(2.12) into the fast roll-slow roll parts.

The potential of eqs. (2.10)-(2.12) contains two parts: $V_{s}$ and $V_{s b}$ where $V_{s}$ is part of the potential that preserves the shift symmetry and $V_{s b}$ is the part that breaks the shift symmetry. Eqs. (2.10)-(2.12) contain a mixture of fast roll and slow roll parts. We wish to decompose eqs. (2.10)-(2.12) to extract the slow roll part. There are $2 m$ number of axionic fields $a_{1}, \cdots, a_{m}$ and $\bar{a}_{1}, \cdots, \bar{a}_{m}$. Since there is only one $\mathrm{U}(1)$ shift symmetry, we can pick a basis where only one linear combination of it is variant under the shift symmetry and all others are invariant. We label this new basis $b_{-}, b_{+}, b_{1}, b_{2}, \cdots, b_{m-1}, \bar{b}_{1}, \bar{b}_{2}, \cdots, \bar{b}_{m-1}$ where only $b_{-}$is sensitive to the shift symmetry. An explicit exhibition of this basis is below

$$
\begin{aligned}
& b_{k}=\frac{a_{k+1}}{f_{k+1}}-\frac{a_{1}}{f_{1}}, \quad k=1,2, \cdots, \quad m-1, \\
& \bar{b}_{k}=\frac{\bar{a}_{k+1}}{\bar{f}_{k+1}}-\frac{\bar{a}_{1}}{\bar{f}_{1}}, \quad k=1,2, \cdots, \quad m-1, \\
& b_{+}=\frac{a_{1}}{f_{1}}+\frac{\bar{a}_{1}}{\bar{f}_{1}}, \\
& b_{-}=\frac{1}{\sqrt{\sum_{k=1}^{m} f_{k}^{2}+\sum_{k=1}^{m} \bar{f}_{k}^{2}}}\left(\sum_{k=1}^{m} f_{k} a_{k}-\sum_{k=1}^{m} \bar{f}_{k} \bar{a}_{k}\right) .
\end{aligned}
$$

Thus the first three equations in eq. (3.1) give us $2(m-1)+1=2 m-1$ linear combinations of axionic fields which are invariant under the shift symmetry while the last one gives us the combination of axionic fields which is sensitive to shift symmetry. It can be easily checked that $b_{-}$is orthogonal to all the rest, i.e.,

$$
\left(b_{-}, b_{k}\right)=0=\left(b_{-}, \bar{b}_{k}\right), \quad\left(b_{-}, b_{+}\right)=0, \quad k=1,2, \cdots, m-1 .
$$


We identify $b_{-}$as the inflaton since in the absence of breaking of the shift symmetry it is massless while the remaining $(2 m-1)$ fields $b_{+}, b_{k}, \bar{b}_{k}, k=1, \cdots, m-1$ are massive. Thus the slow roll is controlled by $b_{-}$only. Accordingly one can decompose the scalar potential into two parts, $V_{\text {fast }}$ and $V_{\text {slow }}$ where

$$
V(b, \bar{b})=V_{\text {fast }}\left(b_{+},\left\{b_{k}\right\},\left\{\bar{b}_{k}\right\}\right)+V_{\text {slow }}\left(b_{-}\right), \quad k=1, \cdots, m-1 .
$$

While both $V_{\text {fast }}$ and $V_{\text {slow }}$ can be computed from eqs. (2.10)-(2.12), here we focus on $V_{\text {slow }}$. The following projections are useful in extracting the slow roll part of the potential

$$
\begin{aligned}
\left(b_{-},(r-1) \frac{a_{k}}{f_{k}}-\frac{\bar{a}_{l}}{\bar{f}_{l}}\right) & =\frac{r}{f_{e}}, & \left(b_{-},(r-1) \frac{\bar{a}_{k}}{\bar{f}_{k}}-\frac{a_{l}}{f_{l}}\right) & =-\frac{r}{f_{e}}, \\
\left(b_{-},(r-2) \frac{a_{k}}{f_{k}}-2 \frac{\bar{a}_{l}}{\bar{f}_{l}}\right) & =\frac{r}{f_{e}}, & \left(b_{-},(r-2) \frac{\bar{a}_{k}}{\bar{f}_{k}}-2 \frac{a_{l}}{f_{l}}\right) & =-\frac{r}{f_{e}}, \\
\left(b_{-},(r-l) \frac{a_{k}}{f_{k}}\right) & =\frac{r-l}{f_{e}}, & \left(b_{-},(r-l) \frac{\bar{a}_{k}}{\bar{f}_{k}}\right) & =\frac{-r+l}{f_{e}},
\end{aligned}
$$

where

$$
f_{e}=\sqrt{\sum_{k=1}^{m} f_{k}^{2}+\sum_{k=1}^{m} \bar{f}_{k}^{2}} .
$$

Using eq. (3.4) in eq. (2.12), and using eq. (2.9) to eliminate $\lambda$, one finds

$$
\begin{aligned}
V_{\text {slow }}\left(b_{-}\right)= & \sum_{k=1}^{m}\left[2 \sum_{r=1}^{q} r\left(A_{k r} f_{k}^{r-1} \sum_{l=1}^{q} l A_{k l} f_{k}^{l-1}+\bar{A}_{k r} \bar{f}_{k}^{r-1} \sum_{l=1}^{q} l \bar{A}_{k l} \bar{f}_{k}^{l-1}\right)\right. \\
& \times\left(1-\cos \left(\frac{r}{f_{e}} b_{-}\right)\right)-2 \sum_{l=1}^{q} \sum_{r=l+1}^{q} l r\left(A_{k l} A_{k r} f_{k}^{l+r-2}+\bar{A}_{k l} \bar{A}_{k r} \bar{f}_{k}^{l+r-2}\right) \\
& \left.\times\left(1-\cos \left(\frac{r-l}{f_{e}} b_{-}\right)\right)\right]
\end{aligned}
$$

A remarkable aspect of eq. (3.6) is that the cosine functions depend only on an effective decay constant $f_{e}$. Thus for the $2 m$ number of fields, if we set $f_{k}=f=\bar{f}_{k}$, we have $f_{e}=\sqrt{(2 m)} f$ which means that even with $f$ sub-Planckian we can get the effective $f_{e}$ much larger than $M_{P l}$ if we choose $m$ large enough. We note that if we set $f_{k}=f=\bar{f}_{k}$, and $N=2 m$, eq. (3.5) takes the form $f_{e}=\sqrt{N} f$ which is similar to what one has in the case of N-flation [24]. However, the inflation potential arising from the fast roll-slow roll splitting is very different from the one in N-flation. The implication of eq. (3.5) and its simplified version $f_{e}=\sqrt{N} f$ is the following: by inclusion of more fields the range of the axion decay constant consistent with inflation is enlarged which increases the allowed parameter space of the theory. Specifically the region of the sub-Planckian domain of the axion decay constant is significantly enlarged. One generally expects this result in the reduction of the type described above, see, e.g., eq. (3.18) of [111]. 


\section{Extension to supergravity}

Next we extend the analysis to supergravity where the scalar potential has the form $[76,77]$

$$
V=e^{K}\left[D_{i} W K_{i j^{*}}^{-1} D_{j^{*}} W^{*}-3|W|^{2}\right]+V_{D} .
$$

Here $K$ is the Kähler potential and $W$, as in global supersymmetry, is the superpotential. Further,

$$
D_{i} W=\frac{\partial W}{\partial \phi_{i}}+\frac{\partial K}{\partial \phi_{i}} W
$$

The $D$-term of the potential, $V_{D}$, will play no role in our analysis and we omit it from here on. Now in supergravity there is the well known $\eta$ problem (see appendix B for the definition of $\eta$ ) which arises because the Kähler potential contributes to $\eta$ and this contribution can be $O(1)$ while for slow roll inflation one needs $\eta \ll 1$. To avoid the $\eta$ problem we will use the following form for the Kähler potential, i.e,

$$
K=\sum_{i} \frac{1}{2}\left(S_{i}+S_{i}^{\dagger}\right)^{2}
$$

We consider here for simplicity a single pair of axions with opposite shift symmetries. We parametrize the complex scalar component $\phi_{i}$ of $S_{i}, i=1,2$ so that

$$
\phi_{i}=\left(\rho_{i}+i a_{i}\right) / \sqrt{2}, i=1,2,
$$

where $a_{i}$ have the shift property

$$
a_{1} \rightarrow a_{1}+\lambda, \quad a_{2} \rightarrow a_{2}-\lambda,
$$

and $\rho_{i}$ are the saxion fields. In this case it is easily checked that the kinetic energy is given by

$$
L_{k i n}=-\frac{1}{2}\left[\partial_{\mu} \rho_{i} \partial^{\mu} \rho_{i}+\partial_{\mu} a_{i} \partial^{\mu} a_{i}\right]
$$

so the fields $a_{i}$ and $\rho_{i}$ are canonically normalized. The superpotential is chosen to be of the form

$$
\begin{aligned}
W & =W_{s}+W_{s b}, \\
W_{s} & =\frac{1}{2} \mu\left(S_{1}+S_{2}\right)^{2}+\frac{1}{3} \lambda\left(S_{1}+S_{2}\right)^{3}+W_{0}, \\
W_{s b} & =P\left(S_{1}\right)+P\left(S_{2}\right),
\end{aligned}
$$

where $W_{0}$ arises from a hidden sector. For supergravity analysis the saxion can be stabilized by imposition of spontaneous symmetry breaking conditions [78] $D_{i} W=0, i=1,2$ which give

$$
\sqrt{2} \mu f+2 \lambda f^{2}+P^{\prime}\left(\frac{f}{\sqrt{2}}\right)+\sqrt{2} f \alpha=0
$$


where

$$
\alpha=\mu f^{2}+\frac{2 \sqrt{2}}{3} \lambda f^{3}+2 P\left(\frac{f}{\sqrt{2}}\right)+W_{0},
$$

while the vanishing of the vacuum energy after the inflationary period gives the additional constraint $\alpha=0$. For shift symmetry breaking we assume $P=\sum_{n} A_{n} e^{c_{n} S}$ so that

$$
W_{s b}=\sum_{n=1}^{q} A_{n}\left(e^{c_{n} S_{1}}+e^{c_{n} S_{2}}\right) .
$$

Next we choose a new basis for the axions, where we replace $a_{1}, a_{2}$ by $b_{+}, b_{-}$so that

$$
b_{ \pm}=\frac{1}{\sqrt{2}}\left(a_{1} \pm a_{2}\right)
$$

where $b_{+}$is invariant under the shift symmetry while $b_{-}$is sensitive to the shift symmetry. As in the global supersymmetry case the computation of the inflation potential is carried out by expanding around the minimum of the saxion potential. Further, we retain only the slow roll part of the potential which involves $b_{-}$. In this case $W_{s b}$ takes the form

$$
W_{s b}=-\sum_{n=1}^{q} B_{n}\left(e^{i \gamma_{n} \frac{b_{-}}{\sqrt{2} f}}+e^{-i \gamma_{n} \frac{b_{-}}{\sqrt{2} f}}\right),
$$

where $B_{n}=-A_{n} e^{c_{n} f / \sqrt{2}}$ and $\gamma_{n}=c_{n} f / \sqrt{2}$. In this case, after stabilization of the saxions and imposition of a vanishing vacuum energy at the end of inflation, and using the fast roll-slow roll splitting mechanism we get the following potential for the inflation field

$$
\begin{aligned}
V\left(b_{-}\right)=e^{2 f^{2}} & {\left[2 \sum_{n=1}^{q} \sum_{m=1}^{q} c_{n} c_{m} B_{n} B_{m}\left(1-2 \cos \left(\frac{\gamma_{n} b_{-}}{\sqrt{2} f}\right)+\cos \left(\left(\gamma_{n}-\gamma_{m}\right) \frac{b_{-}}{\sqrt{2} f}\right)\right)\right.} \\
& +\sum_{n=1}^{q} \sum_{m=1}^{q}\left(16 f^{2}+8 \sqrt{2} f c_{n}-12\right) B_{n} B_{m}\left(1-\cos \left(\gamma_{n} b_{-} / \sqrt{2} f\right)-\cos \left(\gamma_{m} b_{-} / \sqrt{2} f\right)\right. \\
& \left.\left.+\frac{1}{2} \cos \left(\left(\gamma_{n}-\gamma_{m}\right) b_{-} / \sqrt{2} f\right)+\frac{1}{2} \cos \left(\left(\gamma_{n}+\gamma_{m}\right) b_{-} / \sqrt{2} f\right)\right)\right]
\end{aligned}
$$

As noted in the beginning, the analysis above is for one pair of axionic chiral fields. As in the global supersymmetry case the analysis can be extended to $m$ pairs of axionic chiral fields. Again as for the global supersymmetry case this extension would modify the effective axion decay constant for the inflation field so that $\sqrt{2} f$ is replaced by $\sqrt{2 m} f$.

\section{Consistency with Planck data $[14,15]$}

\subsection{Experimental observables}

The path for testing inflationary models with experimental data from the cosmic microwave background (CMB) radiation anisotropies has been well laid out in the literature. The central quantities that enter are the correlation functions involving scalar and tensor perturbations of the inflaton and of the gravitational field which are coupled. From the correlation 
functions one deduces the power spectrum for curvature perturbations and the power spectrum for tensor perturbations and the spectral indices. These are the quantities which are experimentally measured. A brief description of these is given in section B which also defines the notation we use in this section, i.e., $\mathcal{P}_{\mathcal{R}}$ for the power spectrum for comoving curvature perturbations, $\mathcal{P}_{t}$ for the power spectrum for tensor perturbaions, and $n_{s}, n_{t}$ for the scalar and tensor spectral indices. Inflationary models typically exhibit the power law behavior $\mathcal{P}_{\mathcal{R}} \propto k^{n_{s}-1}$ and $\mathcal{P}_{t} \propto k^{n_{t}}$. The power spectrum for the curvature perturbations can be expanded around the pivot scale $k_{0}$, where the pivot scale is the scale chosen for carrying out the analysis, so that

$$
\begin{aligned}
\mathcal{P}_{\mathcal{R}}(k) & =\mathcal{P}_{\mathcal{R}}\left(k_{0}\right)\left(\frac{k}{k_{0}}\right)^{n_{s}(k)-1}, \\
n_{s}(k) & =n_{s}\left(k_{0}\right)+\frac{1}{2}\left(\frac{d n_{s}}{d \ln k}\right) \ln \left(\frac{k}{k_{0}}\right) .
\end{aligned}
$$

Similarly for the tensor perturbations we have

$$
\begin{aligned}
& \mathcal{P}_{t}(k)=\mathcal{P}_{t}\left(k_{0}\right)\left(\frac{k}{k_{0}}\right)^{n_{t}(k)}, \\
& n_{t}(k)=n_{t}\left(k_{0}\right)+\frac{1}{2}\left(\frac{d n_{t}}{d \ln k}\right) \ln \left(\frac{k}{k_{0}}\right) .
\end{aligned}
$$

One also defines a quantity $r$ which is the ratio of the tensor to scalar power spectrum so that

$$
r=\frac{\mathcal{P}_{t}\left(k_{0}\right)}{\mathcal{P}_{\mathcal{R}}\left(k_{0}\right)}
$$

The current experimental limits from Planck experiment at $k_{0}=0.05 \mathrm{Mpc}^{-1}$ are ss follows $[14-16]$

$$
\begin{aligned}
n_{s} & =0.9645 \pm 0.0049(68 \% \mathrm{CL}) \\
r & <0.07(95 \% \mathrm{CL})
\end{aligned}
$$

while $n_{t}\left(k_{0}\right)$ is currently not constrained. As discussed in appendix B the spectral indices can be related to the slow roll parameters $\epsilon$ and $\eta$ (see eq. (B.23)). Here we will focus on the computation of the spectral indices as these are directly measured. Further, one requires the number of e-foldings to be in the range $N_{e}=[50,60]$ where $N_{e}$ is the number of e-foldings between the horizon exit and the end of inflation, i.e., $N_{e}=N_{\text {total }}-N_{\text {exit }}$, where $N_{\text {exit }}$ is the number of e-foldings at horizon exit and $N_{\text {total }}$ is the total number of e-foldings at the end of inflation. For a mode $k$, horizon exit occurs at time when $k=R H=\dot{R}$ and the Hubble radius is $(R H)^{-1}$. To test our model with Planck data we use the code of [80] which is suitable for multi field inflation models. We have checked this code for simple potentials by a direct integration of the Friedman equations for an inflaton field (see appendix A). The code provides the spectral indices $n_{s}, n_{t}$ and the ratio of the tensor to scalar power spectrum. 


\subsection{Monte Carlo analysis for fit to data}

In this subsection we carry out a Monte Carlo analysis on the parameter space of the models we consider. First we consider the model of eq. (3.6). However, we will make some simplifying assumptions in the analysis which are: $A_{k l}=\bar{A}_{k l}=B_{l} f^{3-l}, B_{l}=B G_{l}, f_{k}=f$ for all $k$. Thus $B_{l}, B, G_{l}$ are dimensionless while $f_{k}=f$ carry dimension of mass. Let us take the potential of eq. (3.6) and simplify it using these assumptions which gives

$$
\begin{aligned}
V_{\text {slow }}\left(b_{-}\right)=4 m f^{4} B^{2}( & \sum_{l=1}^{q} l G_{l} \sum_{r=4}^{q} r G_{r}\left(1-\cos \left(\frac{r}{\sqrt{2 m}} \frac{b_{-}}{f}\right)\right) \\
& \left.-\sum_{l=1}^{q} \sum_{r=l+4}^{q} l r G_{l} G_{r}\left(1-\cos \left(\frac{r-l}{\sqrt{2 m}} \frac{b_{-}}{f}\right)\right)\right) .
\end{aligned}
$$

Thus eq. (5.5) is the simplified version of eq. (3.6). We remind the reader that in eq. (5.5), $m$ is the number of axion pairs where the two axions in each pair have opposite $\mathrm{U}(1)$ shift symmetry, and $q$ is the highest power in the polynomial that breaks the shift symmetry. We assume that the terms in the polynomial that break the shift symmetry are operators with dimension higher than 3 in the superpotential. Thus we assume that $r$ takes on the values in the range $[4, q \geq 4]$.

Before embarking on a full Monte Carlo analysis we wish to test the accuracy of the fast roll-slow roll decomposition. As shown in sections 3 and 4, the procedure of decomposing the axion potential into fast roll and slow roll parts brings in a huge simplification in the analysis. We need to verify, however, that neglecting the fast roll part of the potential is a valid approximation. For that we consider a simple case where we compute spectral indices using the full potential including slow roll and fast roll and then just the slow roll by itself. The case we consider is when we have just one pair of axions, i.e., $m=1$, and we consider a single term in the polynomial that breaks the shift symmetry, i.e., we take just the term $G_{4}$. Further we set $G_{4}=1$ since it can be absorbed in the parameter $B$. The analysis is done in high precision to exhibit the difference between the two cases. The example we choose is taken from a Monte Carlo analysis and the model point satisfies the Planck data constraints. However, $f>M_{P l}$ in the example below, but this can be easily alleviated by choosing $m>1$ and the decay constant $f^{\prime}=f / \sqrt{m}$. Here we first run the slow-roll part of the potential. The specific parameters here are $f=14.9605 M_{P l}, b_{-, 0}=13.963 M_{P l}$, and $N_{\text {pivot }}=56$. In this case, $n_{s}=0.953167 \pm 0.000007, n_{t}=-0.005756 \pm 0.000007, r=0.044045984 \pm$ 0.000000003 , where errors were computed by varying the number of subhorizon e-foldings in the integration. Next, we run the full (both slow and fast) simulation with $\mu=M_{P l}$, $B=10^{-4}$, and $a_{1}=\left(b_{-, 0}+13 M_{P l}\right) / \sqrt{2}, \bar{a}_{1}=\left(-b_{-, 0}-13 M_{P l}\right) / \sqrt{2}$. Here one finds that $N_{\text {total }}$ increases from 79.2 to 126.0 due to the presence of the fast roll part. However, the inflation observables in this case are: $n_{s}=0.953169 \pm 0.000006, n_{t}=-0.005754 \pm 0.000006$, and $r=0.044045988 \pm 0.000000010$. As one can see the accuracy of slow-roll fast-roll decomposition surpasses the precision of the integration. We further compare these results to the answers obtained with a slow-roll approximation, from which we get $\epsilon=0.00288$, $\eta=-0.0150, r=0.04604, n_{s}=0.9527, n_{t}=-0.005755$. As one can see apart from $n_{t}$ the discrepancy is now significantly larger than the integration precision. We get $\delta r=0.002$, 

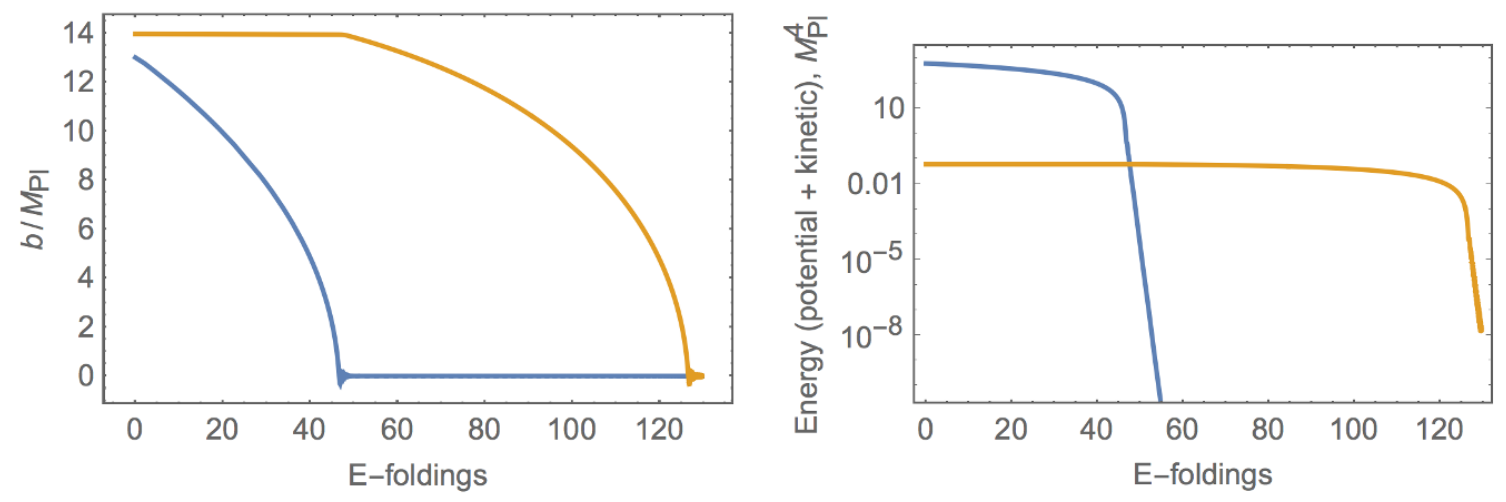

Figure 1. Left panel: fast and slow field components as a function of the e-foldings. Right panel: energy of the slow and fast field components as a function of e-foldings. Slow field energy is defined as $E_{\text {slow }}=V_{\text {slow }}\left(b_{-}\right)+\frac{1}{2}{\dot{b_{-}}}^{2}$. Fast field energy is defined as $E_{\text {fast }}=V_{\text {full }}\left(b_{-}, b_{+}\right)-V_{\text {slow }}\left(b_{-}\right)+\frac{1}{2}{\dot{b_{+}}}^{2}$. One can see that the fast component starts larger, but then the slow component overtakes it. Here $B=10^{-4}$.

$\delta n_{s}=0.0005, \delta n_{t}=0.000001$ comparing to the single-field simulation. However, while the $n_{t}$ value is extremely close to the slow roll prediction, there is a deviation in the value of $r$. Thus more precise measurement of $r$, would allow a test for deviation from the slow roll relation $n_{t}+r / 8=0$.

Similarly, for a supergravity example on figure 7 , we get $r=(1.03994375 \pm 0.00000002) \times$ $10^{-4}, n_{s}=0.9724973 \pm 0.0000007, n_{t}=(-1.29 \pm 0.07) \times 10^{-5}$. On the other hand, from slowroll approximation we get, $r=1.07 \times 10^{-4}, n_{s}=0.9714, n_{t}=-1.34 \times 10^{-5}$. Here, both values of $r$ are way below experimental limit (as is true for most cases on figure 10), $\delta n_{s}=0.0011$, which is $22 \%$ of the experimental limit, and the difference for $n_{t}$ is smaller than the simulations precision.

A comparison of fast roll vs slow roll is given in figure 1. Here the left panel gives the fast and the slow field components as a function of the number of e-foldings. The right panel gives a comparison of the energy of the slow field and the energy of the fast field components as a function of the number of e-foldings. One finds that the fast component starts with a larger energy, but the slow component overtakes it and drives inflation. In figure 2 we give the fast and the slow field components as a function of time in the left panel. On the right panel we display the energy of the slow field and of the fast field as a function of time. We note that the fast field component dies about a factor of 100 faster than the slow component in this case. Similar behavior generally occurs in both global supersymmetry and supergravity models as long as the slow-roll part of the potential is much smaller than the fast-roll part. For that reason we disregard the fast-roll part in the further analysis, and perform simulations with the slow-roll part only.

We proceed now to discuss the result of the full Monte Carlo analysis of the parameter space of eq. (5.5). In the left panel of figure 3 we give an analysis of $r$ vs $n_{s}$ for the 

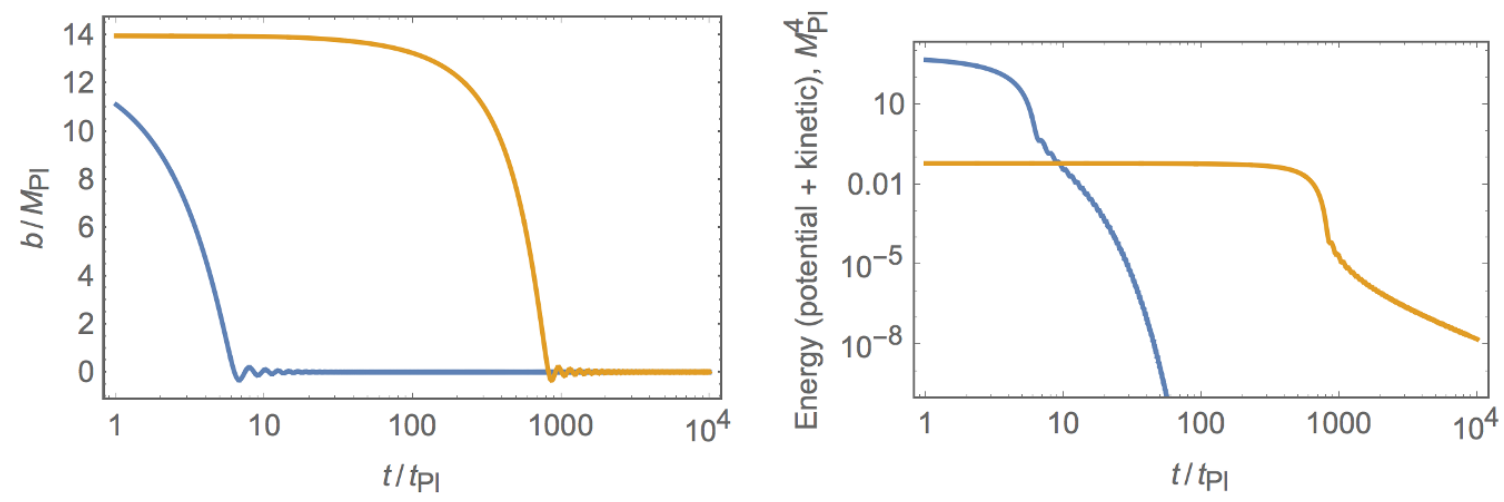

Figure 2. Left panel: fast and slow field components as a function of time. Right panel: energy of the slow and fast field components as a function of time. Slow field energy is defined as $E_{\text {slow }}=$ $V_{\text {slow }}\left(b_{-}\right)+\frac{1}{2} \cdot{ }_{-}^{2}$. Fast field energy is defined as $E_{\text {fast }}=V_{\text {full }}\left(b_{-}, b_{+}\right)-V_{\text {slow }}\left(b_{-}\right)+\frac{1}{2}{\dot{b_{+}}}^{2}$. One can see that the fast component starts larger, but then the slow component overtakes it. Note the logarithmic scale of time. Here $B=10^{-4}$.

parameter space when $G_{4}=1, G_{5} \neq 0$. The scatter plot is on the Planck data where the blue region corresponds to $68 \%$ and $95 \%$ CL regions of Planck TT, TE, EE + lowP in figure 11 of [15]. One finds that there are a large number of parameter points in the region in $r$ vs $n_{s}$ allowed by the Planck experiment. The black dots and the green dots correspond to scenarios where $b_{-}$reaches global minimum at the end of inflation. The scatter points have a mixture of axion decay constant both below and above the Planck mass. In the right panel we exhibit the data set where $f<M_{P l}$. We note in passing that the parameter points in the left panel where $f>M_{P l}$ may be reduced to lie below $M_{P l}$ by choosing the appropriate number of axion pairs $m$.

Next we extend our result to include $G_{6} \neq 0$. The analysis enlarges the number of allowed models which pass all the experimental tests and have $f<M_{P l}$. We do this by starting with the parameter points passing experimental tests from the analysis of figure 3 and extending the region corresponding to these points into $G_{6} \neq 0$ region. The analysis shows that the inflation trajectory is a narrow strip in the $\left(G_{5}, G_{6}\right)$ plane as shown in figure 4 . In figure 5 we give the distribution in $\left(n_{s}, r\right)$ for the parameter space of figure 4 . We note that here $N_{\text {pivot }}=[50,60]$, the full set of the parameter points pass the experimental constraints on $n_{s}$ and $r$ and the parameter points have $f<M_{P l}$. In figure 6 we give the distribution of $n_{t}$ vs. $n_{s}$ for the same parameter space as for figure 5 . Currently there is no experimental data on $n_{t}$ and thus the distribution in the $\left(n_{s}, n_{t}\right)$ plane is a prediction which may be tested in future experiments. Cleary a constraint on $n_{t}$ will narrow down the range of the parameter space further. Here we note that the inflationary trajectory which lies in a narrow strip in the $\left(G_{5}, G_{6}\right)$ plane in figure 4 indicates that inflation with desired properties does not arise for random choices of parameters but occurs along a constrained path in the $\left(G_{5}, G_{6}\right)$ plane. Thus inflation is a fine tuned phenomenon. Part of the reason 

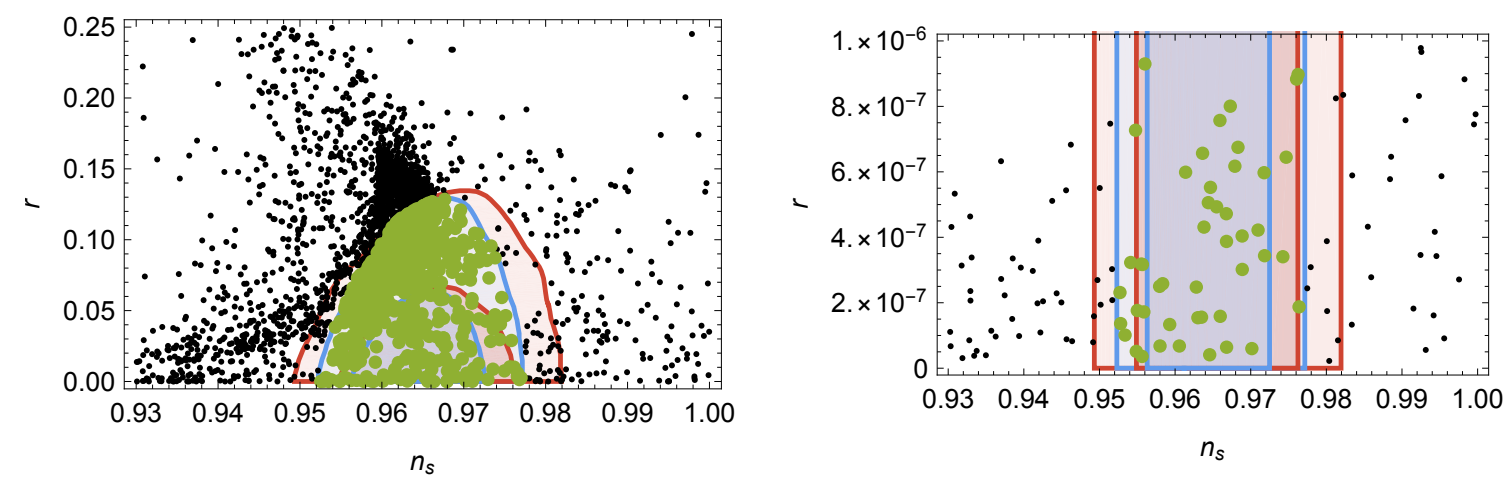

Figure 3. Monte-Carlo analysis for the model with $G_{4}=1, G_{5} \neq 0$ for the entire input parameters space shown in observables space. The blue region corresponds to $68 \%$ (inner contour) and $95 \%$ CL (outer contour) regions of Planck experiment [15] TT, TE, EE + lowP (include polarization data). The red regions correspond to Planck TT+lowP (do not include polarization data). For a discussion of TT, TE, EE + lowP see reference [15]. The green and the black scatter points corresponds to scenarios where $b_{-}$reaches global minimum at the end of inflation, and the green scatter points satisfy experimental bounds on $n_{s}$ and $r$ for the TT, TE, EE + lowP Planck data analysis. All model points have $N=[50,60]$. The decay constant $f<M_{P l}$ for all points on the right panel.

for fine tuning is the following: while inflation with a number of e-foldings can arise for a much larger parameter space, the parameter space gets successively reduced as one imposes the constraint on $N_{\text {pivot }}=[50,60]$, and the constraint on $n_{s}$ and on $r$ (see, e.g., figure 3). In other words one does not expect that any random choice of parameters will result in the desired number of e-foldings and with $n_{s}$ and $r$ consistent with experiment. Thus inflation occurs in small patches of the parameter space but the number of such patches is very large. A similar phenomenon occurs in electroweak symmetry breaking in supergravity models [88] where the constraints of color and charge conservation, relic density constraints and the constraint to have the $Z$-mass to be the experimental value significantly reduce the parameter space of supergravity models. We note in passing that all the parameter points exhibited in figures 3-6 satisfy the Lyth bound [89] for slow roll inflation, i.e.,

$$
\frac{\Delta \phi}{M_{P l}} \geq\left(\frac{r}{0.01}\right)^{1 / 2}
$$

where $\Delta \phi=\left(\phi_{\text {end }}-\phi_{\text {exit }}\right)$. We also note in passing that the inflaton mass in the cases considered above is $O\left(10^{-4}\right) M_{P l}$. With reference to figure 4 we note that the purpose of the analysis is to present one concrete example of a trajectory which supports inflation consistent with data but there could be other trajectories which do the same. The generation of such trajectories is computer intensive and a dedicated computer analysis would be needed to exhaust all the allowed regions where inflation can occur.

Finally we give the Monte Carlo analysis for the supergravity model of eq. (4.13). We begin by displaying in figure 7 a generic potential for $b_{-}$for the supergravity case when $m=1$ (see eq. (4.13)). Next for the same parameter set as used in figure 7 we exhibit the 


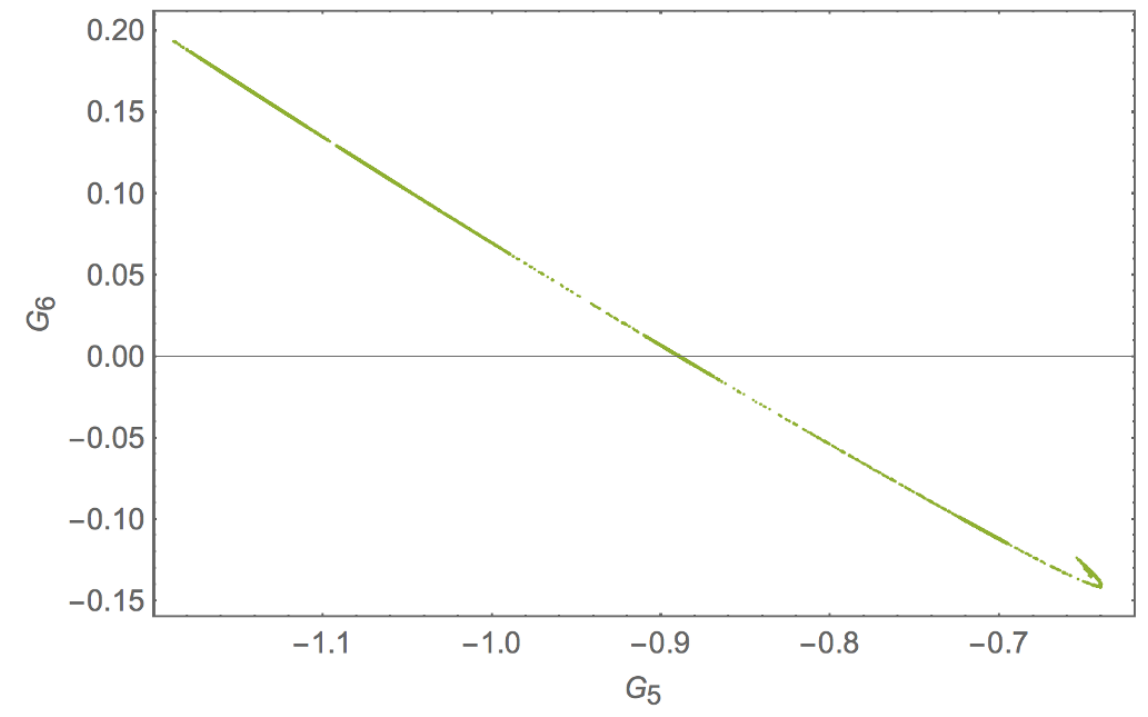

Figure 4. Exhibition of a region in $\left|G_{6}\right|<\left|G_{5}\right|$ plane where all experimental constraints are satisfied. The region exhibited does not exclude other regions where consistent inflation can occur. The analysis shows that inflation can occur in extended regions of the parameter space of models.

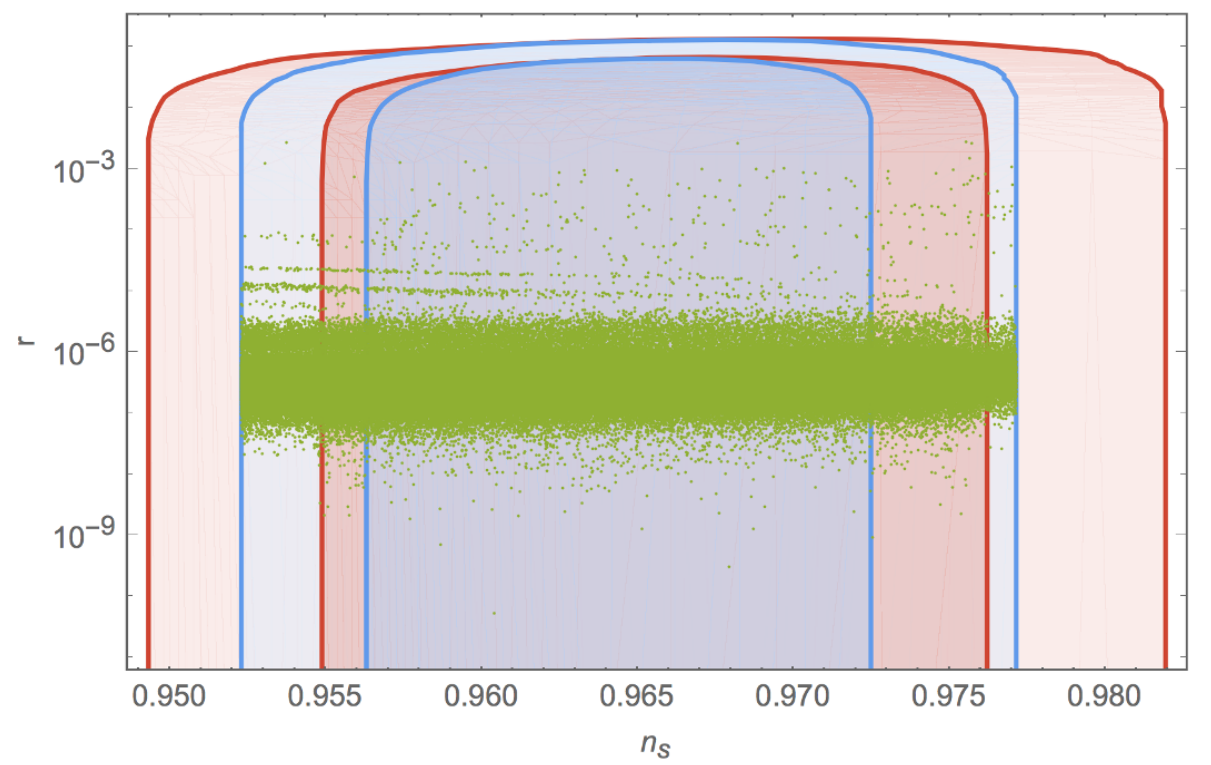

Figure 5. Monte-Carlo analysis when $G_{4}=1, G_{5} \neq 0, G_{6} \neq 0$ for the entire input parameters space shown in $\left(n_{s}, r\right)$ space. The entire set of points shown pass all experimental tests. Note the $\log$ scale on the $r$ axis. 


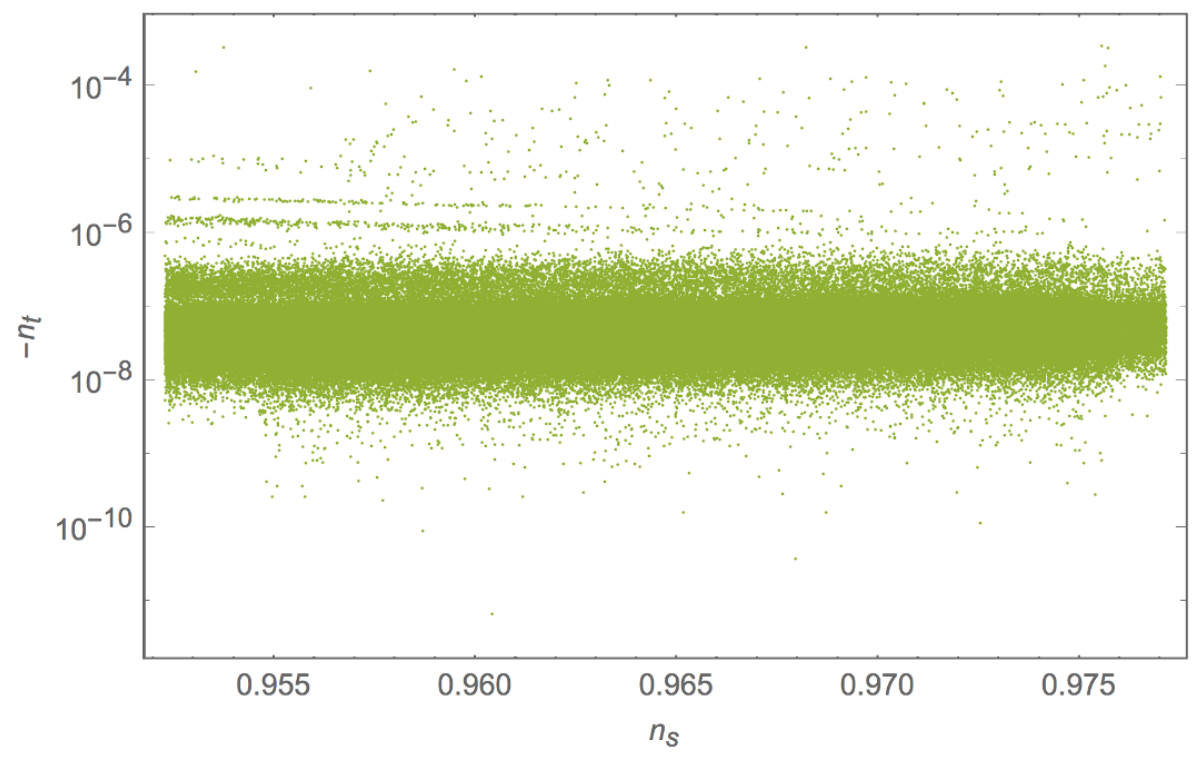

Figure 6. Monte-Carlo analysis when $G_{4}=1, G_{5} \neq 0, G_{6} \neq 0$ for the entire input parameters space shown in $\left(n_{s}, n_{t}\right)$ space for the same dataset as in figure 5 . Note the log scale on the $-n_{t}$ axis.

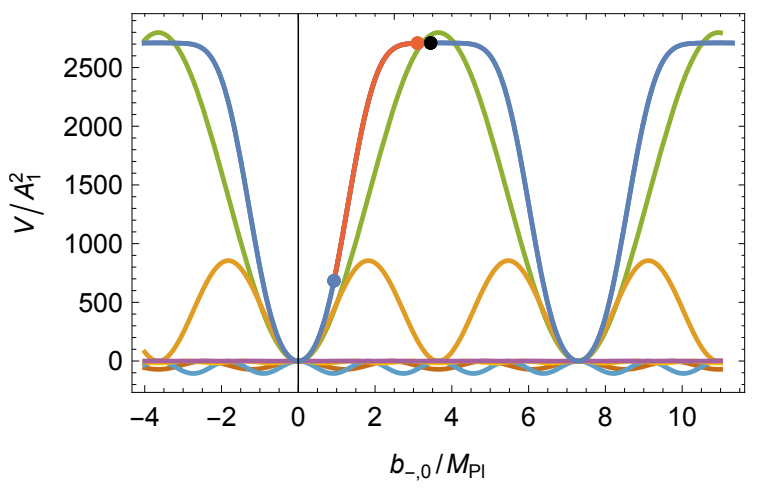

Figure 7. The red and blue curve shows a generic example of a slow-roll potential in $q=3$ supergravity model. Here $A_{2} / A_{1}=8.9588 \times 10^{-2}, A_{3} / A_{1}=4.1713 \times 10^{-3}, \gamma_{k}=k, f / M_{P l}=0.8211$, $b_{-, 0} / M_{P l}=3.448, N_{\text {pivot }}=56.6$. For this case, the observables are $n_{s}=0.9714, n_{t}=-1.34 \times 10^{-5}$, $r=1.07 \times 10^{-4}$. The black dot corresponds to the initial value of the field, the red dot to horizon exit, and the blue dot to the end of inflation. Other curves show contributions to the potential from individual cosine modes. Thus the green curve is plot of the first term in eq. (C.2) in appendix C while the brown curve is plot of the second term in eq. (C.2). The remaining terms in eq. (C.2) are curves with small amplitudes in the plot. 

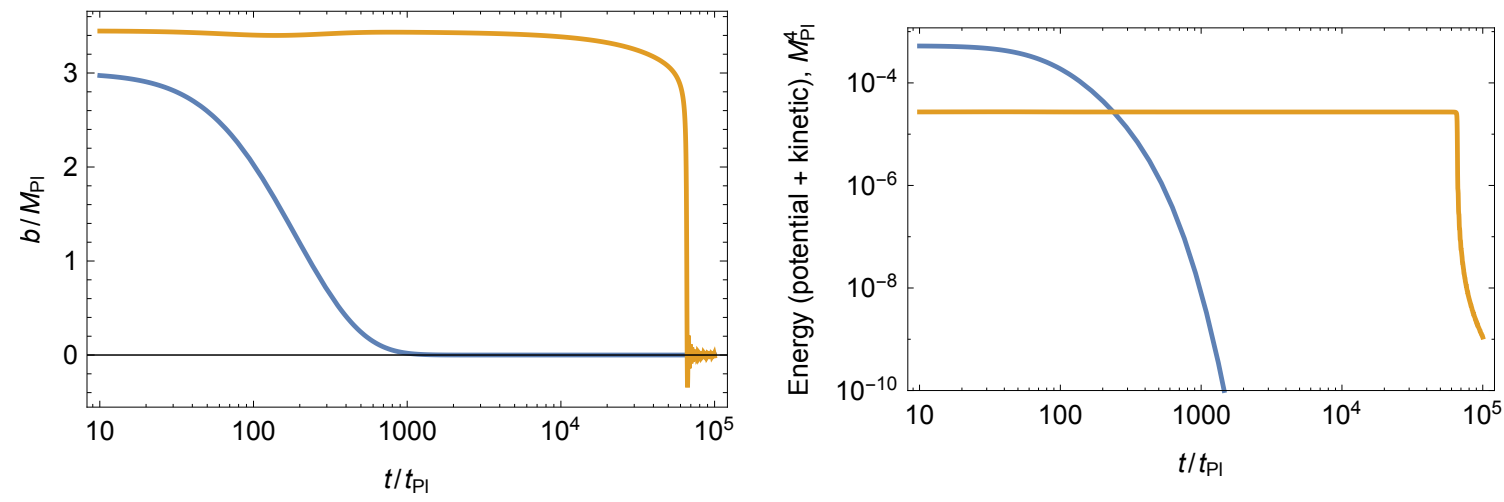

Figure 8. Fast vs. slow field evolution for the case of figure 7. Left panel: fast and slow field components as a function of time. Right panel: energy of the slow and fast field components as a function of time. Slow field energy is defined as $E_{\text {slow }}=V_{\text {slow }}\left(b_{-}\right)+\frac{1}{2} b_{-}{ }^{2}$. Fast field energy is defined as $E_{\text {fast }}=V_{\text {full }}\left(b_{+}, b_{-}\right)-V_{\text {slow }}\left(b_{-}\right)+\frac{1}{2} b_{+}{ }^{2}$. One can see that the fast component starts larger, but then the slow component overtakes it. Note the logarithmic scale of time. Here $A_{1}=10^{-4} M_{P l}^{2}$.
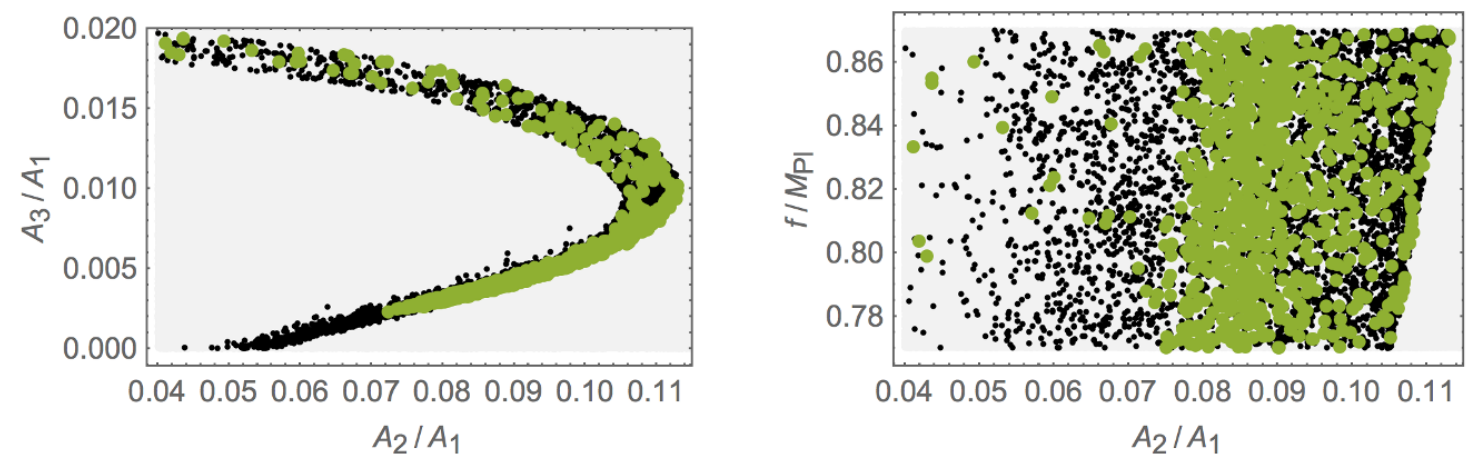

Figure 9. Left panel: $A_{3} / A_{1}$ vs. $A_{2} / A_{1}$ for the experimentally acceptable points for the $q=3$ supergravity model in the input parameters space for the case of one pair of axions. Right panel: the same parameter space plotted in the $f / M_{P l}$ vs $A_{2} / A_{1}$. The green and black parameter points have the same meaning as in figure 3 . Note the entire parameter space of this supergravity model exhibited here has $f<M_{P l}$.

phenomenon of fast roll and slow role in figure 8. Thus in figure 8 the left panel gives the evolution of the fast component (blue) and of the slow component (red) as a function of time, while the right panel gives the energy of the fast (blue) and the slow (red) component as a function of time. As in the global supersymmetry case here too one finds that the fast component dies about a hundred times faster than the slow component and thus the slow component rules inflation. To test the model with experiment, we note that for the case $q=1$, the desired number of e-foldings are not achieved, while for the case $q=2$ one can get the right number of e-foldings but not the desired values of $n_{s}$. However, the 

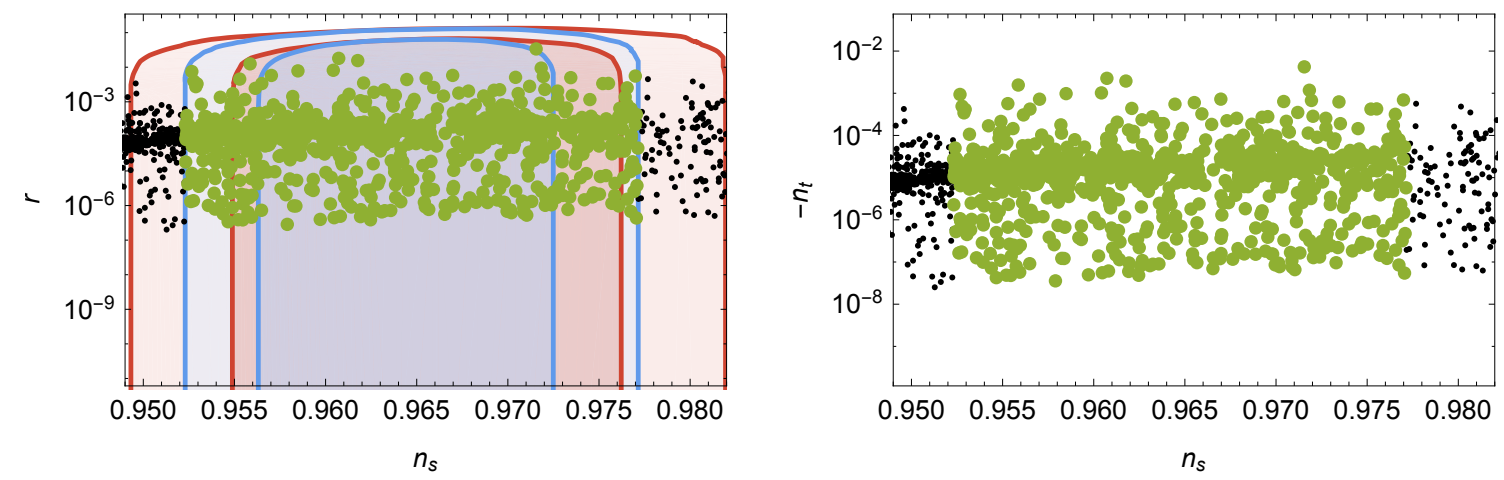

Figure 10. Left panel: display of $r$ vs $n_{s}$ for the same parameter space as in figure 9. Right panel: display $n_{t}$ vs $n_{s}$ for the same parameter space as in figure 9 . The green and black parameter points have the same meaning as in figure 3 .

case $q=3$ gives consistent inflation. Here we investigate the range of the parameter space where $A_{1}=M_{P l}^{2}, A_{2} / A_{1}=[-1,1], A_{3} / A_{2}=[-1,1], f / M_{P l}=[0,1]$. In figure 9 we exhibit the parameter space which gives rise to consistent inflation. The left panel displays the parameter space in the $A_{3} / A_{1}$ vs. $A_{2} / A_{1}$ plane and the right panel exhibits the parameters space in the $f$ vs. $A_{2} / A_{1}$ plane. As can be seen in this panel all the parameter space exhibited has $f$ lying in the sub-Planckian region. In the left panel of figure 10 we give the prediction of $r$ vs $n_{s}$ and in the right panel a prediction of $n_{t}$ vs $n_{s}$. In figures 9 and 10 the green and the black points have the same meaning as in figure 3. As in global supersymmetry case, here too all the parameter points exhibited in figures 9-10 satisfy the Lyth bound [89].

The analysis given above shows that the effective theory does not automatically lead to the exact standard single-field inflation results but the predictions of the model lie very close to them. For example, in one of the cases considered above one finds deviations from the standard single-field case so that $\delta r=0.002, \delta n_{s}=0.0005$ and $\delta n_{t}=0.000001$. Thus as stated earlier although the $n_{t}$ value is very close to the slow roll prediction, there is a deviation in the value of $r$ and a more precise measurement of $r$ would allow a test for deviation from the slow roll relation $n_{t}+r / 8=0$.

\subsection{Generation of a flat inflation potential}

In this section we give further details on the generation of a locally flat scalar potential arising from the instanton induced axion superpotential. In supersymmetric models symmetry breaking terms are added in the superpotential with coefficients which are exponentially suppressed. For $q$ number of symmetry breaking terms induced by instantons in the superpotential, there are in general $n=2 q$ number of cosines with different periods in the scalar potential which follows from the simple relationship between the scalar potential and the superpotential. Further, the coefficients of the cosines contain products of suppression factors because the scalar potential generates cross-terms which automatically arise in going from the superpotential to the scalar potential as can be seen from eq. (38) where a double 
sum appears on the cosines and their coefficients. Such cross terms are relevant in generating a local flatness of the scalar potential. To illustrate this point more clearly we exhibit in appendix $\mathrm{C}$ the analytic expression for the scalar potential for the case $m=1, q=3$ relevant for figure 7 . First, we note that in this case one has six cosines which are of the form $\cos \left(\frac{n b}{\sqrt{2} f}\right), n=1, \cdots, 6$ and we have displayed the suppression factors for each of the six cosines in eq. (72). In eq. (72) $A_{i}, i=1,2,3$ are the suppression factors and we see that products of them appear in all six cosines. Thus $A_{1}$ appears in the first four, $A_{2}$ appears in the first five and $A_{3}$ appears in the coefficients of all six cosines. In eq. (73) we give the numerical sizes of the coefficients of the cosines for the case of figure 7 . Here we note that for the case when

$$
\frac{b}{\sqrt{2} f}=\pi
$$

(which corresponds to $b=3.65$ for $f=0.8211$ used in figure 7) the terms in the first, the third and the fifth brace in eq. (73) give maximum contributions while the contributions of the terms in the second, the fourth and the sixth brace vanish. Thus we see three sets of terms going through a maximum and the other three going through a minimum. With reference to figure 7 , we see that indeed the first cosine term is going through its maximum while the second cosine is going through its minimum at $b=3.65$. The higher cosine terms are following similar patterns although their contributions are relatively small. A superposition of these leads to a local flatness around the point where the maxima and the minima simultaneously occur. This can be roughly seen by just superposing the first two dominant terms in eq. (73) which appear as the green curve and the brown curve in figure 7. Further from eq. (73) one can also understand the size of the potential in the region of flatness.

We note in passing that a similar procedure of using several non-perturbative terms to produce inflation by adjustment of parameters in the non-perturbative terms is used in the so-called 'racetrack' models (see, e.g., [65, 93-106] and for related works [107-110]). In our analysis the shift symmetry produces a flat direction and its breaking reduces the shift symmetry from a continuous to a discrete one. The breaking produces a specific combination of cosines with different periods which leads to local flatness in the potential at points where the maxima of one set of cosines overlaps with the minima of the other set of cosines.

\section{Conclusion}

We have investigated models of multi-field inflation when there is an underlying U(1) shift symmetry. We have proposed a new technique, the fast roll-slow roll splitting mechanism, which involves a decomposition of the inflation potential into two parts: a fast roll part and a slow roll part. The technique was tested and found to be highly accurate. The fast roll-slow roll decomposition reduces the problem of multi-field inflation to that of a single field inflation and is likely to be realized in string theory because of the possible existence of many axions with U(1) symmetry in strings. We have applied this technique for the analysis of spectral indices for an inflation model in a supersymmetric axionic landscape. 
The breaking of the shift symmetry was accomplished by instanton inspired terms in the superpotential. It is found that a large part of the parameter space exists where a number of e-foldings in the range $[50,60]$ are accomplished. This parameter space is reduced on imposing the experimental limit on the spectral index $n_{s}$ of curvature perturbations and the limit on the ratio of the power spectrum of tensor perturbations to the power spectrum of curvature perturbations. Further, in the analysis we show that inflation, which satisfies all experimental constraints, can be achieved with sub-Planckian values of the decay constant which is a significant result of the analysis. It is found that the axion landscape model can reach most of the allowed region of experiment for the spectral indices. The model allows the inflaton field to have initial values which lie in the super-Planckian region and thus there is a possibility of gravitational waves during the inflationary period. Among other topics of interest is the heating after inflation (for a recent review see [90]) and a variety of associated phenomena such as non-Gaussianities in the curvature perturbations, generation of primordial black holes and of primordial magnetic fields, and baryogenesis. These are topics worthy of study within the well defined framework presented in this work which is a model with stabilized saxions and supports inflation consistent with all of the current experimental constraints. Since strings are the most natural framework where axionic landscape can occur, it would be natural to extend this analysis to strings which should be an interesting topic of investigation in the future a realistic analyses could be done with stabilized moduli as in [91, 92].

\section{Acknowledgments}

Conversations with Jim Halverson, Cody Long and Fernando Quevedo are acknowledged. This research was supported in part by the NSF Grant PHY-1620575.

\section{A Preliminaries}

As our starting point we assume homogeneity on cosmological scales, so that the metric takes the form

$$
d s^{2}=-d t^{2}+R(t)^{2}\left(d x^{2}+d y^{2}+d z^{2}\right),
$$

where $R(t)$ is the scale factor. For $2 m$ number of axionic fields, the Lagrangian is given by

$$
\mathcal{L}=-\frac{1}{2} \sum_{a=1}^{2 m}\left(\partial_{\mu} \phi_{a} \partial^{\mu} \phi_{a}\right)-V(\phi) .
$$

In this case the two Friedman equations are

$$
\begin{aligned}
3 M_{P l}^{2} \frac{\dot{R}^{2}}{R^{2}} & =\frac{1}{2} \dot{\phi}_{a} \dot{\phi}_{a}+V(\phi), \\
-2 M_{P l}^{2} \frac{\ddot{R}}{R}-M_{P l}^{2} \frac{\dot{R}^{2}}{R^{2}} & =\frac{1}{2} \dot{\phi}_{a} \dot{\phi}_{a}-V(\phi) .
\end{aligned}
$$

Further the field equations give us

$$
\ddot{\phi_{a}}+3 \frac{\dot{R}}{R} \dot{\phi}_{a}=-\frac{\partial V}{\partial \phi_{a}} .
$$


Let us define the number of e-foldings $N$ so that $N=\ln \left(R / R_{0}\right)$, where $R_{0}$ is the value of $R$ at the beginning of inflation. One can derive the following equations for $N$ from the Friedman equations eqs. (A.2) and the field equation eq. (A.3)

$$
\begin{aligned}
3 M_{P l}^{2} \dot{N}^{2} & =\frac{1}{2} \dot{\phi}_{a} \dot{\phi}_{a}+V(\phi), \\
\ddot{\phi_{a}}+3 \dot{N} \dot{\phi}_{a} & =-\frac{\partial V}{\partial \phi_{a}} .
\end{aligned}
$$

Integration of these equations allows for a determination of the axion fields as a function of the number of e-foldings and also as a function of time.

\section{B Power spectrum and spectral indices}

To test the inflation model with Planck data $[14,15]$ we need to compute the power spectrum of curvature and tensor perturbations and spectral indices. A brief discussion is given here to define notation and make the numerical analysis more comprehensible, and more exhaustive treatments can be found in several reviews [81-84]. Let us begin by considering a massless scalar field within a gravitational background so that

$$
\mathcal{A}_{\phi}=\int d^{4} x \sqrt{-g}\left(-\frac{1}{2} \partial_{\mu} \phi \partial^{\mu} \phi\right) .
$$

It is found convenient to use the conformal time $\zeta$ so that

$$
\zeta=\int \frac{d t}{R(t)} .
$$

Perturbations of the inflaton field should be considered along with the perturbations of the gravitational field. The general linear perturbations of the FRW metric can be parametrized as

$$
d s^{2}=R^{2}\left[-(1+2 \alpha) d \zeta^{2}+2 \beta_{i} d x^{i} d \zeta+\left(\delta_{i j}+h_{i j}\right) d x^{i} d x^{j}\right],
$$

where $\alpha, \beta_{i}, h_{i j}$ parametrize the perturbations. These perturbations can be decomposed into scalar, vector and tensor components. For $\beta_{i}$ we have

$$
\beta_{i}=\nabla_{i} \beta+\beta_{i}^{\prime}, \quad \nabla_{i} \beta^{\prime i}=0,
$$

where $\beta$ is the scalar and $\beta_{i}^{\prime}$ the vector component. We decompose $h_{i j}$ so that

$$
\begin{aligned}
h_{i j} & =2 \gamma \delta_{i j}+2 \nabla_{i} \nabla_{j} \kappa+2 \Delta_{(i} \kappa_{j)}+\kappa_{i j}, \\
\nabla_{i} \kappa^{i j} & =0, \kappa^{i j} \delta_{i j}=0 .
\end{aligned}
$$

First we consider the scalar perturbations. It turns out that the scalar perturbation of interest is given by the combination

$$
\sigma=R\left(\delta \phi-\gamma \frac{\phi^{\prime}}{\mathcal{H}}\right)
$$


where the prime on $\phi$ stands for derivative of $\phi$ with respect to the conformal time $\zeta$ and $\mathcal{H}$ is the Hubble parameter in conformal time. Here $\sigma$ is a combination of the scalar field and metric perturbations and is gauge invariant. The quadratic part of action for $\sigma$ is given by

$$
\mathcal{A}_{\sigma} \simeq \frac{1}{2} \int d \zeta d^{3} x\left[\sigma^{\prime 2}+\vec{\nabla} \sigma \cdot \vec{\nabla} \sigma+\frac{z^{\prime \prime}}{z} \sigma^{2}\right]
$$

where $z=R \phi^{\prime} / \mathcal{H}$. In the slow roll approximation one has $z^{\prime \prime} / z \simeq R^{\prime \prime} / R$. Thus eq. (B.7) takes the form

$$
\mathcal{A}_{\sigma} \simeq \frac{1}{2} \int d \zeta d^{3} x\left[\sigma^{\prime 2}+\vec{\nabla} \sigma \cdot \vec{\nabla} \sigma+\frac{R^{\prime \prime}}{R} \sigma^{2}\right] .
$$

We now do a canonical quantization of the system by imposing the conformal time commutation relations

$$
\begin{aligned}
{\left[\sigma(\zeta, \vec{x}), \pi\left(\zeta, \vec{x}^{\prime}\right)\right] } & =i \hbar \delta\left(\vec{x}-\vec{x}^{\prime}\right), \\
{\left[\sigma(\zeta, \vec{x}), \sigma\left(\zeta, \vec{x}^{\prime}\right)\right] } & =0=\left[\pi(\zeta, \vec{x}), \pi\left(\zeta, \vec{x}^{\prime}\right)\right],
\end{aligned}
$$

where $\pi(\zeta, \vec{x})=\sigma^{\prime}$. A Fourier expansion of $\sigma(\zeta, \vec{x})$ leads to

$$
\sigma(\zeta, \vec{x})=\int \frac{d^{3} k}{(2 \pi)^{3 / 2}}\left[a(\vec{k}) \sigma(\vec{k}, \zeta) e^{i \vec{k} \cdot \vec{x}}+a^{\dagger}(\vec{k}) \sigma^{*}(\vec{k}, \zeta) e^{-i \vec{k} \cdot \vec{x}}\right],
$$

and quantization of the creation and the destruction operators $a(\vec{k})$ and $a(\vec{k})^{\dagger}$ so that

$$
\begin{aligned}
{\left[a(\vec{k}), a^{\dagger}\left(\vec{k}^{\prime}\right)\right] } & =\delta\left(\vec{k}-\vec{k}^{\prime}\right), \\
{\left[a(\vec{k}), a\left(\vec{k}^{\prime}\right)\right] } & =0=\left[a^{\dagger}(\vec{k}), a^{\dagger}\left(\vec{k}^{\prime}\right)\right],
\end{aligned}
$$

where the Fourier component $\sigma(\vec{k}, \zeta)$ obey the equations of motion

$$
\sigma^{\prime \prime}(\vec{k}, \zeta)+\left(k^{2}-\frac{2}{\zeta^{2}}\right) \sigma(\vec{k}, \zeta)=0
$$

In eq. (B.13) we used $R^{\prime \prime} / R=2 / \zeta^{2}$. This result follows from the fact that for the case when $H=\dot{R} / R$ is a constant the scale factor and the conformal time are related so that $R(\zeta)=-(H \zeta)^{-1}$. A specific solution to eq. (B.13) [85-87] is

$$
\sigma(\zeta, \vec{k})=\sqrt{\frac{\hbar}{2 k}}\left(1-\frac{i}{k \zeta}\right) e^{-i k \zeta} .
$$

Using the commutation relations of eq. (B.12) and the result above the correlation function $\langle 0|\sigma(\vec{x}) \sigma(\vec{y})| 0\rangle$ is given by

$$
\langle 0|\sigma(\vec{x}) \sigma(\vec{y})| 0\rangle=\int d^{3} k e^{i \vec{k} \cdot(\vec{x}-\vec{y})} \frac{|\sigma(\zeta, \vec{k})|^{2}}{(2 \pi)^{3}} .
$$

The power spectrum for $\sigma$ is given by

$$
\mathcal{P}_{\sigma}(k)=\frac{k^{3}}{2 \pi^{2}}|\sigma(\zeta, \vec{k})|^{2}
$$


The comoving curvature perturbation $\mathcal{R}$ is defined by

$$
\mathcal{R}=\frac{\mathcal{H}}{\phi^{\prime}} \delta \phi-\gamma
$$

Thus $\mathcal{R}=-\sigma / z$ snd the power spectrum for the curvature perturbation $\mathcal{P}_{\mathcal{R}}(k)$ is given by

$$
\mathcal{P}_{\mathcal{R}}(k)=\frac{k^{3}}{2 \pi^{2}} z^{-2}|\sigma(\zeta, \vec{k})|^{2} .
$$

Now for the case when the wavelength is larger than the Hubble radius one has $k|\zeta| \ll 1$. Further using the result $R(\zeta)=-(H \zeta)^{-1}$ and eq. (B.14) in eq. (B.18) and reverting to the cosmic time rather than using the conformal time one gets

$$
\mathcal{P}_{\mathcal{R}} \simeq \frac{\hbar}{4 \pi^{2}}\left(H^{4} / \dot{\phi}^{2}\right)_{k=R H} .
$$

Computation of the tensor power spectrum is very similar to the scalar case except for the polarization and one gets

$$
\mathcal{P}_{t}=\frac{2 \hbar}{\pi^{2}}\left(\frac{H}{M_{P l}}\right)_{k=R H}^{2} .
$$

$\mathcal{P}_{\mathcal{R}}$ and $\mathcal{P}_{t}$ can be exhibited in terms of the inflaton potential. Here one finds

$$
\begin{aligned}
\mathcal{P}_{\mathcal{R}} & =\frac{1}{12 \pi^{2}}\left(\frac{V^{3}}{M_{P l}^{6} V^{\prime 2}}\right)_{k=R H}, \\
\mathcal{P}_{t} & =\frac{2}{3 \pi^{2}}\left(\frac{V}{M_{P l}^{4}}\right)_{k=R H} .
\end{aligned}
$$

Models of inflation are often parametrized in terms of the so called slow roll parameters $\epsilon, \eta$ defined by

$$
\epsilon=\frac{1}{2}\left(\frac{M_{P l} V^{\prime}}{V}\right)^{2}, \quad \eta=\left|\frac{M_{P l}^{2} V^{\prime \prime}(\phi)}{V(\phi)}\right| .
$$

The spectral indices $n_{s}$ and $n_{t}$ (see eqs. (5.1) and (5.2)) are related to them by

$$
n_{s}=1-6 \epsilon+2 \eta, \quad n_{t}=-2 \epsilon, \quad r=16 \epsilon
$$

where $r$ is defined by eq. (5.3). Eq. (B.23) is for the standard single-field inflation and gives the prediction $n_{t}=-r / 8$. Small deviations from this result can occur for the effective single-field case and the size of the deviations will depend on the specifics of the inputs. A more detailed discussion of this is given in section 5.2.

\section{Emergence of a flat inflation potential for axions}

Here we give an illustration of how a flat inflation potential for axions arises from a superposition of cosine functions with the specific case of figure 7 in mind. To exhibit this we 
begin by simplifying eq. (4.13) for the case of figure 7, i.e., $q=3$, and $\gamma_{k}=k$ for $k=1,2,3$. Setting $M_{P l}=1$, we get:

$$
\begin{aligned}
V_{3}(b)=- & \frac{1}{f^{2}}\left(4 e ^ { 2 f ^ { 2 } + 2 } \left(-2 A_{1}^{2}\left(4 f^{4}+f^{2}+1\right)-e A_{1}\left(A_{2}\left(4 f^{2}+3\right) f^{2}+2 e A_{3}\left(4 f^{4}+5 f^{2}+3\right)\right)\right.\right. \\
& \left.\left.+e^{3} A_{2} A_{3}\left(4 f^{4}+7 f^{2}+12\right)\right)\right)\left(1-\cos \left(\frac{b}{\sqrt{2} f}\right)\right) \\
+ & \frac{1}{f^{2}}\left(2 e ^ { 2 f ^ { 2 } + 2 } \left(A_{1}^{2}\left(-\left(4 f^{4}+f^{2}\right)\right)+2 e A_{1}\left(A_{2}\left(8 f^{4}+6 f^{2}+4\right)-e A_{3}\left(4 f^{4}+5 f^{2}+6\right)\right)\right.\right. \\
& \left.\left.+4 e^{2} A_{2}\left(A_{2}\left(4 f^{4}+5 f^{2}+4\right)+e A_{3}\left(4 f^{4}+7 f^{2}+6\right)\right)\right)\right)\left(1-\cos \left(\frac{2 b}{\sqrt{2} f}\right)\right) \\
+ & \frac{1}{f^{2}}\left(4 e ^ { 2 f ^ { 2 } + 3 } \left(A_{1}\left(2 e A_{3}\left(4 f^{4}+5 f^{2}+3\right)-A_{2} f^{2}\left(4 f^{2}+3\right)\right)\right.\right. \\
& \left.\left.\quad+2 e^{2} A_{3}\left(A_{2}\left(4 f^{4}+7 f^{2}+6\right)+e A_{3}\left(4 f^{4}+9 f^{2}+9\right)\right)\right)\right)\left(1-\cos \left(\frac{3 b}{\sqrt{2} f}\right)\right) \\
- & 2\left(A_{2}^{2}+2 A_{1} A_{3}\right) e^{2 f^{2}+4}\left(4 f^{2}+5\right)\left(1-\cos \left(\frac{4 b}{\sqrt{2} f}\right)\right) \\
- & 4 A_{2} A_{3} e^{2 f^{2}+5}\left(4 f^{2}+7\right)\left(1-\cos \left(\frac{5 b}{\sqrt{2} f}\right)\right) \\
- & 2 A_{3}^{2} e^{2 f^{2}+6}\left(4 f^{2}+9\right)\left(1-\cos \left(\frac{6 b}{\sqrt{2} f}\right)\right) .
\end{aligned}
$$

Substituting $f=0.8211, A_{2} / A_{1}=8.9588 \times 10^{-2}, A_{3} / A_{1}=4.1713 \times 10^{-3}$, as in figure 7 , we obtain:

$$
\begin{aligned}
V_{3}(b) / A_{1}^{2}= & 1398.96\left(1-\cos \left(\frac{b}{\sqrt{2} f}\right)\right)+427.466\left(1-\cos \left(\frac{2 b}{\sqrt{2} f}\right)\right) \\
& -35.4939\left(1-\cos \left(\frac{3 b}{\sqrt{2} f}\right)\right)-52.9837\left(1-\cos \left(\frac{4 b}{\sqrt{2} f}\right)\right) \\
& -8.28504\left(1-\cos \left(\frac{5 b}{\sqrt{2} f}\right)\right)-0.632442\left(1-\cos \left(\frac{6 b}{\sqrt{2} f}\right)\right) .
\end{aligned}
$$

Eq. (C.2) is the potential used in the analysis of figure 7 .

Open Access. This article is distributed under the terms of the Creative Commons Attribution License (CC-BY 4.0), which permits any use, distribution and reproduction in any medium, provided the original author(s) and source are credited.

\section{References}

[1] A.H. Guth, The Inflationary Universe: A Possible Solution to the Horizon and Flatness Problems, Phys. Rev. D 23 (1981) 347 [InSPIRE].

[2] A.A. Starobinsky, A New Type of Isotropic Cosmological Models Without Singularity, Phys. Lett. B 91 (1980) 99 [INSPIRE].

[3] A.D. Linde, A New Inflationary Universe Scenario: A Possible Solution of the Horizon, Flatness, Homogeneity, Isotropy and Primordial Monopole Problems, Phys. Lett. B 108 (1982) 389 [INSPIRE]. 
[4] A. Albrecht and P.J. Steinhardt, Cosmology for Grand Unified Theories with Radiatively Induced Symmetry Breaking, Phys. Rev. Lett. 48 (1982) 1220 [INSPIRE].

[5] K. Sato, First Order Phase Transition of a Vacuum and Expansion of the Universe, Mon. Not. Roy. Astron. Soc. 195 (1981) 467 [INSPIRE].

[6] A.D. Linde, Chaotic Inflation, Phys. Lett. B 129 (1983) 177 [inSPIRE].

[7] A.D. Linde, Particle physics and inflationary cosmology, Contemp. Concepts Phys. 5 (1990) 1 [hep-th/0503203] [INSPIRE].

[8] C. Cheung, P. Creminelli, A.L. Fitzpatrick, J. Kaplan and L. Senatore, The Effective Field Theory of Inflation, JHEP 03 (2008) 014 [arXiv: 0709. 0293] [INSPIRE].

[9] V.F. Mukhanov and G.V. Chibisov, Quantum Fluctuations and a Nonsingular Universe, JETP Lett. 33 (1981) 532 [INSPIRE].

[10] S.W. Hawking, The Development of Irregularities in a Single Bubble Inflationary Universe, Phys. Lett. B 115 (1982) 295 [INSPIRE].

[11] A.A. Starobinsky, Dynamics of Phase Transition in the New Inflationary Universe Scenario and Generation of Perturbations, Phys. Lett. B 117 (1982) 175 [INSPIRE].

[12] A.H. Guth and S.Y. Pi, Fluctuations in the New Inflationary Universe, Phys. Rev. Lett. 49 (1982) 1110 [INSPIRE].

[13] J.M. Bardeen, P.J. Steinhardt and M.S. Turner, Spontaneous Creation of Almost Scale Free Density Perturbations in an Inflationary Universe, Phys. Rev. D 28 (1983) 679 [INSPIRE].

[14] Planck collaboration, R. Adam et al., Planck 2015 results. I. Overview of products and scientific results, Astron. Astrophys. 594 (2016) A1 [arXiv: 1502.01582] [INSPIRE].

[15] Planck collaboration, P.A.R. Ade et al., Planck 2015 results. XX. Constraints on inflation, Astron. Astrophys. 594 (2016) A20 [arXiv:1502.02114] [INSPIRE].

[16] BICEP2 and KeCK Array collaborations, P.A.R. Ade et al., Improved Constraints on Cosmology and Foregrounds from BICEP2 and Keck Array Cosmic Microwave Background Data with Inclusion of 95 GHz Band, Phys. Rev. Lett. 116 (2016) 031302 [arXiv: 1510.09217] [INSPIRE].

[17] K. Freese, J.A. Frieman and A.V. Olinto, Natural inflation with pseudo-Nambu-Goldstone bosons, Phys. Rev. Lett. 65 (1990) 3233 [INSPIRE].

[18] F.C. Adams, J.R. Bond, K. Freese, J.A. Frieman and A.V. Olinto, Natural inflation: Particle physics models, power law spectra for large scale structure and constraints from COBE, Phys. Rev. D 47 (1993) 426 [hep-ph/9207245] [INSPIRE].

[19] T. Banks, M. Dine, P.J. Fox and E. Gorbatov, On the possibility of large axion decay constants, JCAP 06 (2003) 001 [hep-th/0303252] [INSPIRE].

[20] P. Svrček and E. Witten, Axions In String Theory, JHEP 06 (2006) 051 [hep-th/0605206] [INSPIRE].

[21] J.E. Kim, H.P. Nilles and M. Peloso, Completing natural inflation, JCAP 01 (2005) 005 [hep-ph/0409138] [INSPIRE].

[22] C. Long, L. McAllister and P. McGuirk, Aligned Natural Inflation in String Theory, Phys. Rev. D 90 (2014) 023501 [arXiv: 1404.7852] [InSPIRE].

[23] M. Berg, E. Pajer and S. Sjors, Dante's Inferno, Phys. Rev. D 81 (2010) 103535 [arXiv: 0912.1341] [INSPIRE]. 
[24] S. Dimopoulos, S. Kachru, J. McGreevy and J.G. Wacker, N-flation, JCAP 08 (2008) 003 [hep-th/0507205] [INSPIRE].

[25] R. Easther and L. McAllister, Random matrices and the spectrum of N-flation, JCAP 05 (2006) 018 [hep-th/0512102] [INSPIRE].

[26] T.W. Grimm, Axion inflation in type-II string theory, Phys. Rev. D 77 (2008) 126007 [arXiv:0710.3883] [INSPIRE].

[27] R. Kallosh, N. Sivanandam and M. Soroush, Axion Inflation and Gravity Waves in String Theory, Phys. Rev. D 77 (2008) 043501 [arXiv:0710.3429] [INSPIRE].

[28] M.E. Olsson, Inflation assisted by heterotic axions, JCAP 04 (2007) 019 [hep-th/0702109] [INSPIRE].

[29] D. Battefeld and T. Battefeld, Non-Gaussianities in N-flation, JCAP 05 (2007) 012 [hep-th/0703012] [INSPIRE].

[30] S.A. Kim, A.R. Liddle and D. Seery, Non-Gaussianity in axion N-flation models: detailed predictions and mass spectra, Phys. Rev. D 85 (2012) 023532 [arXiv:1108.2944] [INSPIRE].

[31] S.A. Kim, A.R. Liddle and D. Seery, Non-Gaussianity in axion Nflation models, Phys. Rev. Lett. 105 (2010) 181302 [arXiv: 1005.4410] [INSPIRE].

[32] T. Rudelius, On the Possibility of Large Axion Moduli Spaces, JCAP 04 (2015) 049 [arXiv: 1409.5793] [INSPIRE].

[33] N. Arkani-Hamed, H.-C. Cheng, P. Creminelli and L. Randall, Pseudonatural inflation, JCAP 07 (2003) 003 [hep-th/0302034] [INSPIRE].

[34] D.E. Kaplan and N.J. Weiner, Little inflatons and gauge inflation, JCAP 02 (2004) 005 [hep-ph/0302014] [INSPIRE].

[35] D. Green, B. Horn, L. Senatore and E. Silverstein, Trapped Inflation, Phys. Rev. D 80 (2009) 063533 [arXiv:0902.1006] [inSPIRE].

[36] A. Arvanitaki, S. Dimopoulos, S. Dubovsky, N. Kaloper and J. March-Russell, String Axiverse, Phys. Rev. D 81 (2010) 123530 [arXiv:0905.4720] [InSPIRE].

[37] E. Pajer and M. Peloso, A review of Axion Inflation in the era of Planck, Class. Quant. Grav. 30 (2013) 214002 [arXiv: 1305.3557] [INSPIRE].

[38] D.J.E. Marsh, Axion Cosmology, Phys. Rept. 643 (2016) 1 [arXiv:1510.07633] [InSPIRE].

[39] P. Nath, Supersymmetry, Supergravity, and Unification, Cambridge Monographs On Mathematical Physics, Cambridge University Press, Cambridge, U.K. (2016).

[40] J.R. Ellis, D.V. Nanopoulos, K.A. Olive and K. Tamvakis, Primordial supersymmetric inflation, Nucl. Phys. B 221 (1983) 524 [inSPIRE].

[41] H. Murayama, H. Suzuki, T. Yanagida and J. Yokoyama, Chaotic inflation and baryogenesis in supergravity, Phys. Rev. D 50 (1994) R2356 [hep-ph/9311326] [INSPIRE].

[42] E.J. Copeland, A.R. Liddle, D.H. Lyth, E.D. Stewart and D. Wands, False vacuum inflation with Einstein gravity, Phys. Rev. D 49 (1994) 6410 [astro-ph/9401011] [INSPIRE].

[43] E.D. Stewart, Inflation, supergravity and superstrings, Phys. Rev. D 51 (1995) 6847 [hep-ph/9405389] [INSPIRE].

[44] G.R. Dvali, Q. Shafi and R.K. Schaefer, Large scale structure and supersymmetric inflation without fine tuning, Phys. Rev. Lett. 73 (1994) 1886 [hep-ph/9406319] [INSPIRE].

[45] P. Binetruy and G.R. Dvali, D term inflation, Phys. Lett. B 388 (1996) 241 [hep-ph/9606342] [INSPIRE]. 
[46] B. Körs and P. Nath, Hierarchically split supersymmetry with Fayet-Iliopoulos D-terms in string theory, Nucl. Phys. B 711 (2005) 112 [hep-th/0411201] [INSPIRE].

[47] M. Yamaguchi, Supergravity based inflation models: a review, Class. Quant. Grav. 28 (2011) 103001 [arXiv:1101.2488] [inSPIRE].

[48] M. Kawasaki, M. Yamaguchi and T. Yanagida, Natural chaotic inflation in supergravity, Phys. Rev. Lett. 85 (2000) 3572 [hep-ph/0004243] [INSPIRE].

[49] M. Yamaguchi and J. Yokoyama, New inflation in supergravity with a chaotic initial condition, Phys. Rev. D 63 (2001) 043506 [hep-ph/0007021] [INSPIRE].

[50] D. Baumann and D. Green, Signatures of Supersymmetry from the Early Universe, Phys. Rev. D 85 (2012) 103520 [arXiv:1109.0292] [InSPIRE].

[51] M. Yamaguchi, Natural double inflation in supergravity, Phys. Rev. D 64 (2001) 063502 [hep-ph/0103045] [INSPIRE].

[52] M. Kawasaki and M. Yamaguchi, A supersymmetric topological inflation model, Phys. Rev. D 65 (2002) 103518 [hep-ph/0112093] [INSPIRE].

[53] M. Yamaguchi and J. Yokoyama, Chaotic hybrid new inflation in supergravity with a running spectral index, Phys. Rev. D 68 (2003) 123520 [hep-ph/0307373] [INSPIRE].

[54] P. Brax and J. Martin, Shift symmetry and inflation in supergravity, Phys. Rev. D 72 (2005) 023518 [hep-th/0504168] [INSPIRE].

[55] R. Kallosh, On inflation in string theory, Lect. Notes Phys. 738 (2008) 119 [hep-th/0702059] [INSPIRE].

[56] S.C. Davis and M. Postma, SUGRA chaotic inflation and moduli stabilisation, JCAP 03 (2008) 015 [arXiv:0801.4696] [INSPIRE].

[57] F. Takahashi, Linear Inflation from Running Kinetic Term in Supergravity, Phys. Lett. B 693 (2010) 140 [arXiv:1006.2801] [INSPIRE].

[58] K. Nakayama and F. Takahashi, Running Kinetic Inflation, JCAP 11 (2010) 009 [arXiv: 1008.2956] [INSPIRE].

[59] R. Kallosh and A. Linde, New models of chaotic inflation in supergravity, JCAP 11 (2010) 011 [arXiv: 1008.3375] [INSPIRE].

[60] M. Kawasaki, N. Kitajima and K. Nakayama, Cosmological Aspects of Inflation in a Supersymmetric Axion Model, Phys. Rev. D 83 (2011) 123521 [arXiv:1104.1262] [INSPIRE].

[61] J. Ellis, D.V. Nanopoulos and K.A. Olive, No-Scale Supergravity Realization of the Starobinsky Model of Inflation, Phys. Rev. Lett. 111 (2013) 111301 [Erratum ibid. 111 (2013) 129902] [arXiv:1305.1247] [INSPIRE].

[62] M. Czerny, T. Higaki and F. Takahashi, Multi-Natural Inflation in Supergravity, JHEP 05 (2014) 144 [arXiv: 1403.0410] [INSPIRE].

[63] T. Kobayashi, S. Uemura and J. Yamamoto, Polyinstanton axion inflation, Phys. Rev. D 96 (2017) 026007 [arXiv: 1705.04088] [INSPIRE].

[64] N. Okada and Q. Shafi, Gravity Waves, Axion and Gravitino Dark Matter in $\mu$-Hybrid Inflation, arXiv: 1709.04610 [INSPIRE].

[65] J.J. Blanco-Pillado et al., Racetrack inflation, JHEP 11 (2004) 063 [hep-th/0406230] [INSPIRE]. 
[66] S. Krippendorf and F. Quevedo, Metastable SUSY Breaking, de Sitter Moduli Stabilisation and Kähler Moduli Inflation, JHEP 11 (2009) 039 [arXiv:0901.0683] [INSPIRE].

[67] M. Cicoli, K. Dutta, A. Maharana and F. Quevedo, Moduli Vacuum Misalignment and Precise Predictions in String Inflation, JCAP 08 (2016) 006 [arXiv: 1604.08512] [InSPIRE].

[68] R.D. Peccei and H.R. Quinn, Constraints Imposed by CP Conservation in the Presence of Instantons, Phys. Rev. D 16 (1977) 1791 [inSPIRE].

[69] S. Weinberg, A New Light Boson?, Phys. Rev. Lett. 40 (1978) 223 [inSPIRE].

[70] F. Wilczek, Problem of Strong $p$ and $t$ Invariance in the Presence of Instantons, Phys. Rev. Lett. 40 (1978) 279 [INSPIRE].

[71] J. Halverson, C. Long and P. Nath, Ultralight axion in supersymmetry and strings and cosmology at small scales, Phys. Rev. D 96 (2017) 056025 [arXiv:1703.07779] [InSPIRE].

[72] M. Cvetič, J. Halverson and R. Richter, Mass Hierarchies from MSSM Orientifold Compactifications, JHEP 07 (2010) 005 [arXiv:0909.4292] [INSPIRE].

[73] Y. Nambu and G. Jona-Lasinio, Dynamical Model of Elementary Particles Based on an Analogy with Superconductivity. 1., Phys. Rev. 122 (1961) 345 [InSPIRE].

[74] J. Goldstone, Field Theories with Superconductor Solutions, Nuovo Cim. 19 (1961) 154 [INSPIRE].

[75] J. Goldstone, A. Salam and S. Weinberg, Broken Symmetries, Phys. Rev. 127 (1962) 965 [INSPIRE].

[76] A.H. Chamseddine, R.L. Arnowitt and P. Nath, Locally Supersymmetric Grand Unification, Phys. Rev. Lett. 49 (1982) 970 [INSPIRE].

[77] E. Cremmer, S. Ferrara, L. Girardello and A. Van Proeyen, Yang-Mills Theories with Local Supersymmetry: Lagrangian, Transformation Laws and SuperHiggs Effect, Nucl. Phys. B 212 (1983) 413 [inSPIRE].

[78] P. Nath, R.L. Arnowitt and A.H. Chamseddine, Gauge Hierarchy in Supergravity Guts, Nucl. Phys. B 227 (1983) 121 [InSPIRE].

[79] S.M. Leach, A.R. Liddle, J. Martin and D.J. Schwarz, Cosmological parameter estimation and the inflationary cosmology, Phys. Rev. D 66 (2002) 023515 [astro-ph/0202094] [INSPIRE].

[80] M. Dias, J. Frazer and D. Seery, Computing observables in curved multifield models of inflation - A guide (with code) to the transport method, JCAP 12 (2015) 030 [arXiv: 1502.03125] [INSPIRE].

[81] D.H. Lyth and A. Riotto, Particle physics models of inflation and the cosmological density perturbation, Phys. Rept. 314 (1999) 1 [hep-ph/9807278] [INSPIRE].

[82] A.R. Liddle and D.H. Lyth, Cosmological inflation and large scale structure, Cambridge University Press, Cambridge, U.K., (2000).

[83] D. Langlois, Lectures on inflation and cosmological perturbations, Lect. Notes Phys. 800 (2010) 1 [arXiv:1001.5259] [INSPIRE].

[84] D. Baumann, Inflation, in Physics of the large and the small, TASI 09, proceedings of the Theoretical Advanced Study Institute in Elementary Particle Physics, Boulder, Colorado, U.S.A., 1-26 June 2009, pp. 523-686, arXiv:0907.5424, [INSPIRE].

[85] T.S. Bunch and P.C.W. Davies, Quantum Field Theory in de Sitter Space: Renormalization by Point Splitting, Proc. Roy. Soc. Lond. A 360 (1978) 117. 
[86] N.A. Chernikov and E.A. Tagirov, Quantum theory of scalar fields in de Sitter space-time, Ann. Inst. H. Poincare Phys. Theor. A 9 (1968) 109.

[87] C. Schomblond and P. Spindel, Unicity Conditions of the Scalar Field Propagator Delta(1) $(x, y)$ in de Sitter Universe, Ann. Inst. H. Poincare Phys. Theor. 25 (1976) 67.

[88] R.L. Arnowitt and P. Nath, SUSY mass spectrum in SU(5) supergravity grand unification, Phys. Rev. Lett. 69 (1992) 725 [INSPIRE].

[89] D.H. Lyth, What would we learn by detecting a gravitational wave signal in the cosmic microwave background anisotropy?, Phys. Rev. Lett. 78 (1997) 1861 [hep-ph/9606387] [INSPIRE].

[90] M.A. Amin, M.P. Hertzberg, D.I. Kaiser and J. Karouby, Nonperturbative Dynamics Of Reheating After Inflation: A Review, Int. J. Mod. Phys. D 24 (2014) 1530003 [arXiv:1410.3808] [INSPIRE].

[91] S. Kachru, R. Kallosh, A.D. Linde and S.P. Trivedi, de Sitter vacua in string theory, Phys. Rev. D 68 (2003) 046005 [hep-th/0301240] [INSPIRE].

[92] V. Balasubramanian, P. Berglund, J.P. Conlon and F. Quevedo, Systematics of moduli stabilisation in Calabi-Yau flux compactifications, JHEP 03 (2005) 007 [hep-th/0502058] [INSPIRE].

[93] Z. Lalak, G.G. Ross and S. Sarkar, Racetrack inflation and assisted moduli stabilisation, Nucl. Phys. B 766 (2007) 1 [hep-th/0503178] [INSPIRE].

[94] B. Greene and A. Weltman, An effect of $\alpha^{\prime}$ corrections on racetrack inflation, JHEP 03 (2006) 035 [hep-th/0512135] [INSPIRE].

[95] J.J. Blanco-Pillado et al., Inflating in a better racetrack, JHEP 09 (2006) 002 [hep-th/0603129] [INSPIRE].

[96] C.-Y. Sun and D.-H. Zhang, The Non-Gaussianity of Racetrack Inflation Models, Commun. Theor. Phys. 48 (2007) 189 [astro-ph/0604298] [INSPIRE].

[97] P. Brax, A.-C. Davis, S.C. Davis, R. Jeannerot and M. Postma, D-term Uplifted Racetrack Inflation, JCAP 01 (2008) 008 [arXiv:0710.4876] [INSPIRE].

[98] W.-Y. Wen, Inflation in a refined racetrack, arXiv:0712.0458 [INSPIRE].

[99] P. Brax, S.C. Davis and M. Postma, The robustness of $n_{s} \lesssim 0.95$ in racetrack inflation, JCAP 02 (2008) 020 [arXiv: 0712.0535] [INSPIRE].

[100] W.-Y. Wen, Effects of open string moduli on racetrack inflation, Mod. Phys. Lett. A 23 (2008) 1589 [INSPIRE].

[101] P. Brax, C. van de Bruck, A.-C. Davis, S.C. Davis, R. Jeannerot and M. Postma, Racetrack Inflation and Cosmic Strings, JCAP 07 (2008) 018 [arXiv:0805.1171] [INSPIRE].

[102] H.-Y. Chen, L.-Y. Hung and G. Shiu, Inflation on an Open Racetrack, JHEP 03 (2009) 083 [arXiv: 0901.0267] [INSPIRE].

[103] M. Badziak and M. Olechowski, Inflation with racetrack superpotential and matter field, JCAP 02 (2010) 026 [arXiv:0911.1213] [INSPIRE].

[104] R. Allahverdi, B. Dutta and K. Sinha, Low-scale Inflation and Supersymmetry Breaking in Racetrack Models, Phys. Rev. D 81 (2010) 083538 [arXiv:0912.2324] [INSPIRE].

[105] M. Olechowski, Inflation with racetrack superpotential and matter field, J. Phys. Conf. Ser. 259 (2010) 012028 [INSPIRE]. 
[106] M. Badziak, F-term uplifted racetrack inflation, in Proceedings, 45th Rencontres de Moriond on Cosmology: La Thuile, Italy, March 13-20, 2010, pp. 265-268, 2010, arXiv:1005.5537, [INSPIRE].

[107] T. Higaki and F. Takahashi, Natural and Multi-Natural Inflation in Axion Landscape, JHEP 07 (2014) 074 [arXiv: 1404.6923] [INSPIRE].

[108] T. Higaki and F. Takahashi, Axion Landscape and Natural Inflation, Phys. Lett. B 744 (2015) 153 [arXiv: 1409.8409] [InSPIRE].

[109] K. Kadota, T. Kobayashi, A. Oikawa, N. Omoto, H. Otsuka and T.H. Tatsuishi, Small field axion inflation with sub-Planckian decay constant, JCAP 10 (2016) 013 [arXiv: 1606. 03219] [INSPIRE].

[110] T. Kobayashi, A. Oikawa, N. Omoto, H. Otsuka and I. Saga, Constraints on small-field axion inflation, Phys. Rev. D 95 (2017) 063514 [arXiv: 1609. 05624] [INSPIRE].

[111] A. Ernst, A. Ringwald and C. Tamarit, Axion Predictions in $\mathrm{SO}(10) \times \mathrm{U}(1)_{\mathrm{PQ}}$ Models, arXiv:1801.04906 [INSPIRE]. 